\title{
Effectiveness and Safety of Compound Chinese Medicine plus Routine Western Medicine in In-Stent Restenosis: A Meta-Analysis and Systematic Review
}

\author{
Lu Liu $\mathbb{D}^{1,2}$ Jing Liu, ${ }^{1,2}$ Qun Gao, ${ }^{1,2}$ Yang Wu, ${ }^{1,2}$ Jinjin Lu, \\ Jie Wan, 1,3 Yan Li, ${ }^{1,2}$ Xiaoyun Cui,, ${ }^{1,2}$ Kun Zhou, ${ }^{1,4}$ Wenhao Jia,, \\ Yanchao Huang, ${ }^{1,2}$ Wenbai Qu, ${ }^{1,2}$ and Qian Lin (DD ${ }^{1,2}$ \\ ${ }^{1}$ Beijing University of Chinese Medicine, North 3rd Ring East Road, Beijing 100029, China \\ ${ }^{2}$ Department of Cardiology, Dongfang Hospital, Beijing University of Chinese Medicine, Fanggu Road, Beijing 100078, China \\ ${ }^{3}$ Intensive Care Unit, Dongfang Hospital, Beijing University of Chinese Medicine, Fanggu Road, Beijing 100078, China \\ ${ }^{4}$ Scientific Research Division, Dongfang Hospital, Beijing University of Chinese Medicine, Fanggu Road, Beijing 100078, China \\ Correspondence should be addressed to Qian Lin; 13910565673@126.com
}

Received 29 October 2017; Accepted 28 March 2018; Published 11 July 2018

Academic Editor: Kieran Cooley

Copyright (C) $2018 \mathrm{Lu}$ Liu et al. This is an open access article distributed under the Creative Commons Attribution License, which permits unrestricted use, distribution, and reproduction in any medium, provided the original work is properly cited.

Objective. To examine the effects and safety of oral compound Chinese medicine (CCM) plus routine western medicine (RWM) in in-stent restenosis (ISR). Methods. Various electronic databases (CBM, CNKI, VIP, Wanfang, PubMed, EMBASE, and Cochrane Library) were searched until April 2017. The quality of the included studies was evaluated, and meta-analyses were performed using RevMan5.3 and STATA 12.0 software. Moreover, funnel plot and Egger's publication bias plots were analysed to identify publication bias and adverse reactions were reported. A sensitive analysis was carried out according to the quality score. Results. In all, 40 RCTs involving 4536 patients were selected for this review. The pooled estimates of three studies showed that the benefit to the number of ISRs (NoR) was more substantial for CCM plus RWM than for RWM alone (RR 0.24, 95\% CI 0.10 to $0.57, P=0.001 ; I^{2}=0 \%$, $P=0.81$ ). The rate of ISR was significantly lower for CCM plus RWM than for the same RWM alone (RR $0.44,95 \%$ CI 0.37 to $\left.0.53, P<0.00001 ; I^{2}=0 \%, P=0.95\right)$. CCM plus RWM benefitted the rate of ISR when a CM placebo plus RWM was used as the control intervention (RR $0.34,95 \%$ CI 0.20 to $0.57, P<0.0001 ; I^{2}=0 \%, P=0.95$ ). The difference of adverse reactions was not significant. For secondary outcomes, the CCM plus RWM group did not reduce the rates of revascularization and cardiac death, but it did reduce the rate of recurrent angina over the results observed in the RWM alone group. In addition, funnel plot and Egger's publication bias plot indicated that there was publication bias. The association between the use of CCM plus RWM and RWM alone remained significant after the sensitivity analysis excluding studies with low quality score (quality score $\leqslant 4$ ) with a pooled RR of 0.41 (95\% CI, 0.34-0.50). Conclusion. Oral CCM plus RWM clearly benefitted patients with percutaneous coronary intervention (PCI) because it prevented and treated ISR better than was observed for either RWM alone or a CM placebo plus RWM.

\section{Introduction}

Currently, percutaneous coronary intervention (PCI) is widely used around the world to treat coronary artery disease (CAD) and has significantly reduced mortality in patients with acute coronary syndromes [1]. However, the incidence rate of in-stent restenosis (ISR) is still approximately $10 \%$, even accounting for the introduction of drug-eluting stents (DES) [2], and reaches as high as $40 \%-50 \%$ in patients with multivessel involvement [3]. ISR therefore inflicts a heavy burden on both the lives of patients with CAD and the economy and remains a challenge to the implantation of PCI and $\mathrm{CAD}$ prognoses.

In traditional Chinese medicine (TCM), the typical symptoms of ISR, such as chest pain and chest tightness, are referred to as "Xiongbi" [4]. TCM presents mature theories and is supported by abundant clinical experience for treating "Xiongbi". In recent years, the efficacy and safety of TCM in 
ISR has been widely studied with remarkable results. Studies have shown that TCM not only clearly alleviates the typical symptoms of ISR but also improves its long-term prognosis, suggesting that TCM may lead to promising applications for the treatment of ISR.

Previous reviews focused on this issue were published in 2008 [5], 2012 [6], and 2014 [7]. The first two of these studies were systematic reviews (SRs), and the latter was a metaanalysis. They explored the effects of many types of PCI and included studies with small sample sizes (fewer than 30 cases) and that explored a single TCM herb. In addition, a presearch showed that, in the 3 years since 2014, more studies have supported the efficacy and safety of oral compound Chinese medicines (CCMs) in ISR. In this SR, we applied more rigorous inclusion and exclusion criteria and aimed to explore whether oral CCM plus routine western medicine (RWM) is effective and safe for treating ISR after stent implantation. We use CCM because it has wider applications in clinical practice and conforms better to TCM theory.

\section{Methods}

This SR was performed according to the Preferred Reporting Items for Systematic Reviews and Meta-Analyses (PRISMA) Statement [8] and the Cochrane Handbook [9] Systematic review and is registered at PROSPERO with the registration number CRD42017075368 (https://www.crd.york.ac.uk/ PROSPERO).

2.1. Search Strategy. A comprehensive search of 7 medical databases, including the PubMed, EMBASE, Cochrane Library, China National Knowledge Infrastructure (CNKI), Chinese Scientific Journals (VIP), Wanfang, and Chinese Biomedical (CBM) databases, was conducted through April 2017 without a language restriction. We performed the search using individually or combined Mesh terms for all fields relating to the patients (coronary heart disease and coronary artery disease) and interventions (traditional Chinese medicine, Chinese herbal medicine, compound Chinese medicine, Chinese prepared medicine, Chinese herbal formula, and Chinese herbs) of interest. All titles/subjects related to the outcome (in-stent restenosis) were searched. When searching Chinese databases, the above terms were searched in Chinese.

2.2. Inclusion Criteria. Only randomized controlled trials (RCTs) were included. We focused on trials with participants diagnosed with major angiographic criteria-documented [10] CAD who were eligible for stent implantation (with either bare metal stents or drug-eluting stents) regardless of their gender, age, disease course, comorbidity, and ethnic origin. The baseline characteristics of each study were consistent. The interventional treatment was any oral CCM plus RWM administered for at least 1 month regardless of dosage. The control group was treated with the same RWM or with a CM placebo plus the same RWM. "CCM" included Chinese prepared medicines or Chinese herbal formulas. The compositions of the CCM were detailed. Our primary outcomes were restenosis occurring within at least 6 months of followup. Coronary in-stent restenosis is classically defined as the angiographic detection of a recurrent stenosis with a diameter greater than $50 \%$ at the stent segment or the $5 \mathrm{~mm}$ segments adjacent to it (in-segment restenosis). The drop-out rate for coronary angiography (CA) review was required to be lower than $20 \%$. Each included study was required to report the primary outcome. Secondary outcomes could be major adverse cardiac events (MACE), including recurrent angina, myocardial infarction, revascularization, and cardiac death. Only secondary outcomes that improved in equal to or more than $50 \%$ of cases were considered responses. In addition, adverse reactions were considered as a primary safety outcome. When there were several follow-up points, only the last one was considered.

2.3. Exclusion Criteria. Studies with a small sample study (less than 30 cases), duplicate reports, and pilot studies were excluded. Any trial that failed to satisfy the inclusion criteria or for which required data were unavailable was excluded.

2.4. Data Extraction. Two reviewers (Jinjin Lu and Yan Li) independently extracted the data using a standardized extraction form. Disagreements were resolved by census or consultation with a third reviewer (Wenhao Jia). The following items were extracted: study name, year of publication, sample size, details of the trial design (i.e., randomization, allocation concealment, and blinding), eligibility criteria, general characteristics of patients, details of intervention and control therapies, details related to outcomes, details related to drop-outs, and other information that may help detect bias.

2.5. Quality Assessment. Two reviewers (Xiaoyun Cui and Kun Zhou) independently assessed the methodological quality of the included studies using the Cochrane Collaboration's tool [11], which is a domain-based evaluation tool used to generate a "risk of bias" table for each study. Any disagreement was resolved by consensus or consultation with a third reviewer (Yanchao Huang). The domains used for assessment were sequence generation (selection bias), allocation concealment (selection bias), blinding of participants and personnel (performance bias), blinding of outcome assessment (detection bias), incomplete outcome data (attrition bias), selective outcome reporting (reporting bias), and other potential sources of bias (e.g., early termination, contamination, and conflict of interest). Because it is difficult to blind participants in studies of Chinese herbal medicines, performance bias was likely present in all trials. With regard to the objective outcomes adopted in this SR, such as ISR and MACE, performance bias and detection bias might not be as important, and we therefore summarized these trials as low-risk. In addition, with regard to selective outcomes, this domain was ranked as "low-risk" unless the outcomes were critical to our issue.

2.6. Data Analysis. Data synthesis and analysis were performed with RevMan software 5.3 and STATA 12.0 software (StataCorp LP, College Station, TX) [9]. Dichotomous data 


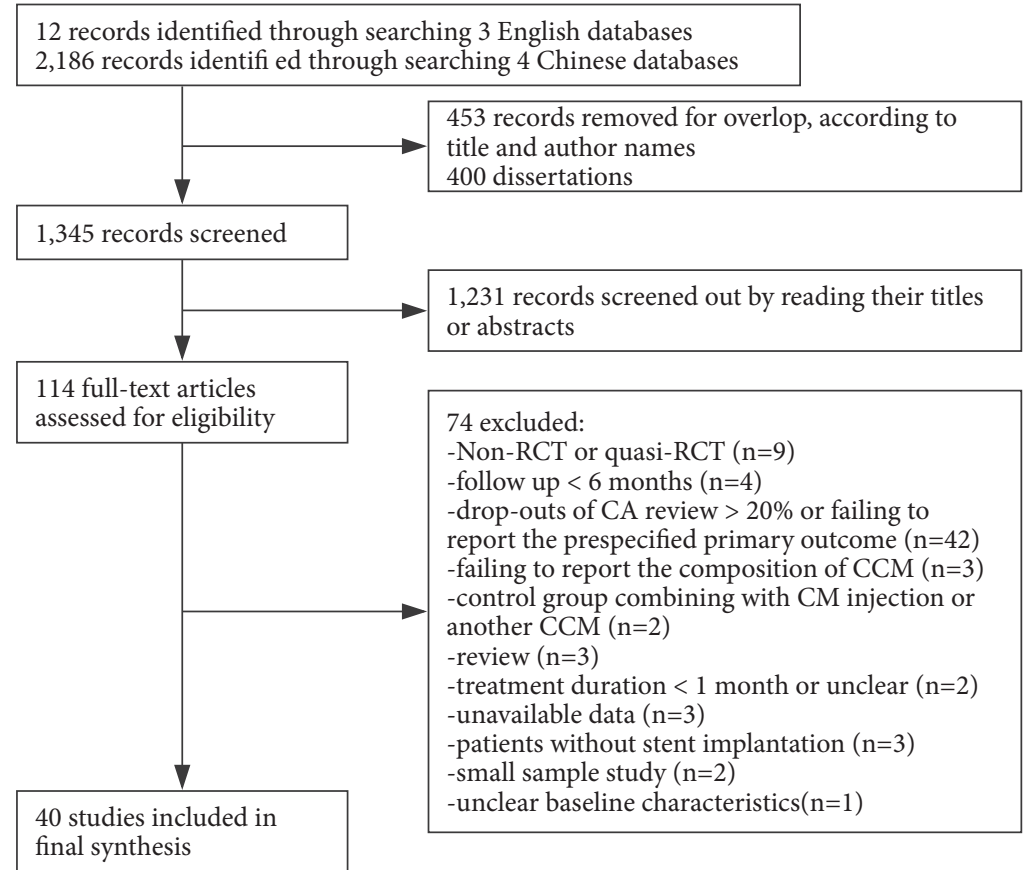

Figure 1: Study flow diagram.

were measured as relative risk (RR), while continuous data were measured as the mean difference (MD), both with corresponding 95\% confidence intervals (CIs). Heterogeneity across trials was measured with the Cochran $Q$ test and is presented as $I^{2}$ statistics. Only trials with $I^{2}$ lower than $85 \%$ were used for the meta-analysis, and the characteristics of the included trials were similar. A fixed effect model was used if $I^{2}$ was lower than $25 \%$. Otherwise, a random effect model was applied under the assumption that any heterogeneity was readily explainable. Furthermore, funnel plot and Egger's test were performed to detect heterogeneity and publication bias, respectively, if sufficient sources were available. A 2-tailed $P$ value less than 0.05 was considered significant. A sensitivity analysis was conducted to assess the stability of the results.

\section{Results}

3.1. Study Identification. All eligible studies were screened and identified (Figure 1). A total of 2198 records were retrieved. Of these, full-text evaluations were conducted on 114 studies. In all, 74 of these 114 studies were excluded for the following reasons: non-RCT or quasi-RCT $(n=9)$; followup $<6$ months $(n=4)$; drop-outs from CA review $>20 \%$ or failure to report the prespecified primary outcome $(n=42)$; failure to report the composition of the CCM $(n=3)$; control group consisted of a CM injection or another CCM $(n=2)$; review $(n=3)$; treatment duration $<1$ month or unclear $(n=2)$; unavailable data $(n=3)$; patients without stent implantation $(n=3)$; small study sample size $(n=2)$; and unclear baseline characteristics $(n=1)$. Finally, a total of 40 RCTs with a total of 4536 patients were included in this SR [12-51].
3.2. Characteristics of Included RCTs. All studies were conducted in China from 2010 to 2017. The average age of the patients ranged from 52.93 to 69.27 years old. Most trials had more males than females. The diagnostic criteria for CAD were mainly based on CA criteria or the Nomenclature and Diagnostic Criteria for Ischemic Heart Disease (World Health Organization, WHO) [52]. All patients successfully underwent stent implantation. The distributions of baseline characteristics were basically the same in each group. Four trials $[25,26,36,49]$ were double-blinded, and, in three trials, the control group used a CM placebo plus RWM [19, 25, 26]. The remaining trials were designed to compare a CCM plus RWM group to a group treated with same RWM alone. Of the 40 studies, 20 used a decoction, 1 used an oral liquid, 2 used granules, 4 used pills, 12 used capsules, and 1 used tablets. RWM mainly consisted of aspirin, Clopidogrel, angiotensinconverting inhibitor (ACEI)/angiotensin II receptor blocker (ARB), beta-blockers, and statins. The dosage and types of RWM were prescribed according to the recommendations of Chinese Society of Cardiology Guideline [10]. Overall, 33 kinds of CCM were used, and the treatment courses ranged from 3 to 12 months with a follow-up time of 6 to 18 months. For outcomes, ISR was assessed by computed tomography angiography (CTA) or CA in all trials; adverse reactions caused by CCMs were reported in 11 studies but were significant in only 2 studies $[26,38]$; MACE were reported in 12 studies and included recurrent angina $(n=12)$, myocardial infarction $(n=3)$, revascularization $(n=2)$, and cardiac death $(n=5)$ (Tables 1 and 2).

3.3. Methodological Quality of Included RCTs. Of the 40 included studies, 13 were randomized by random number 


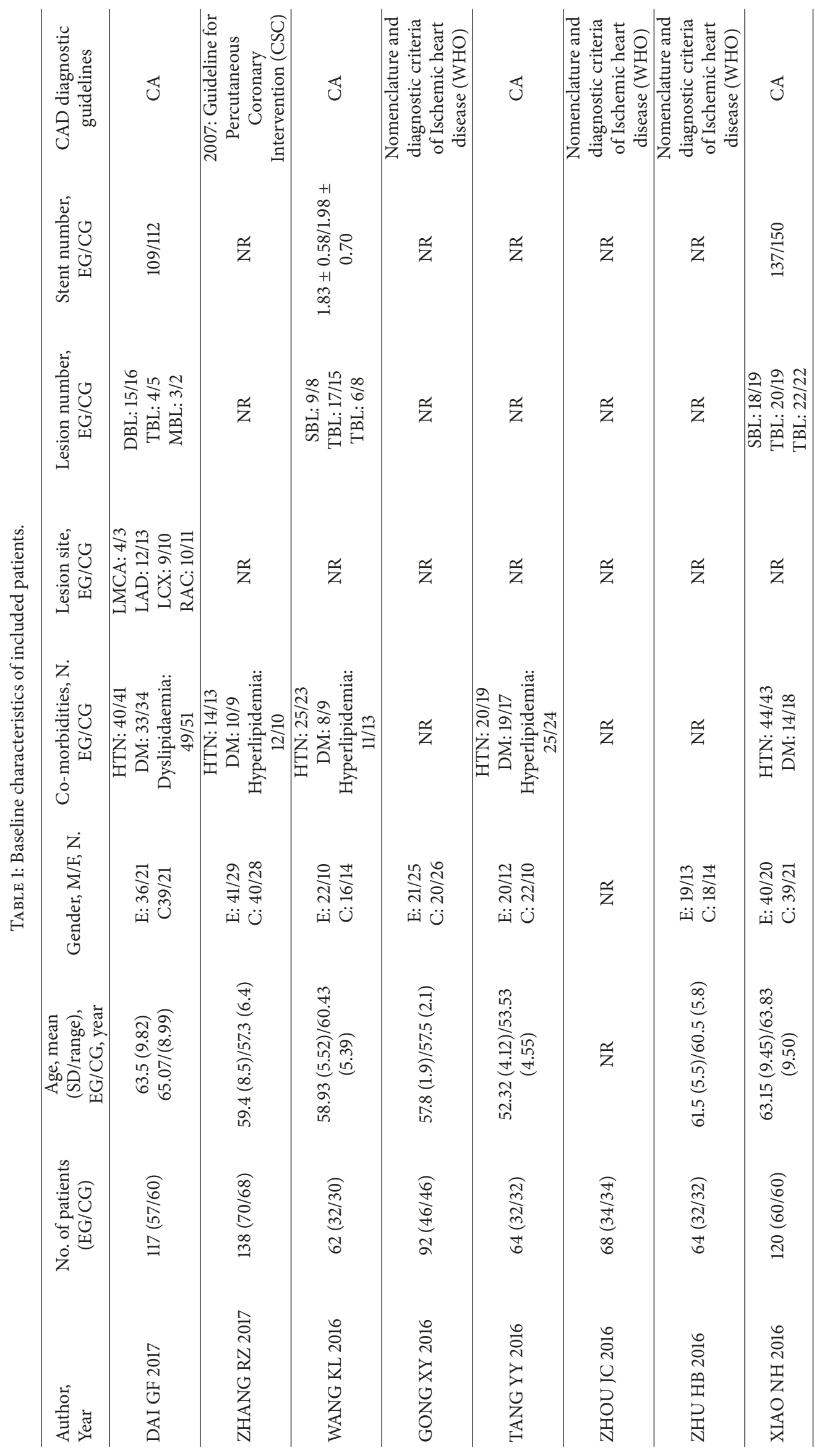




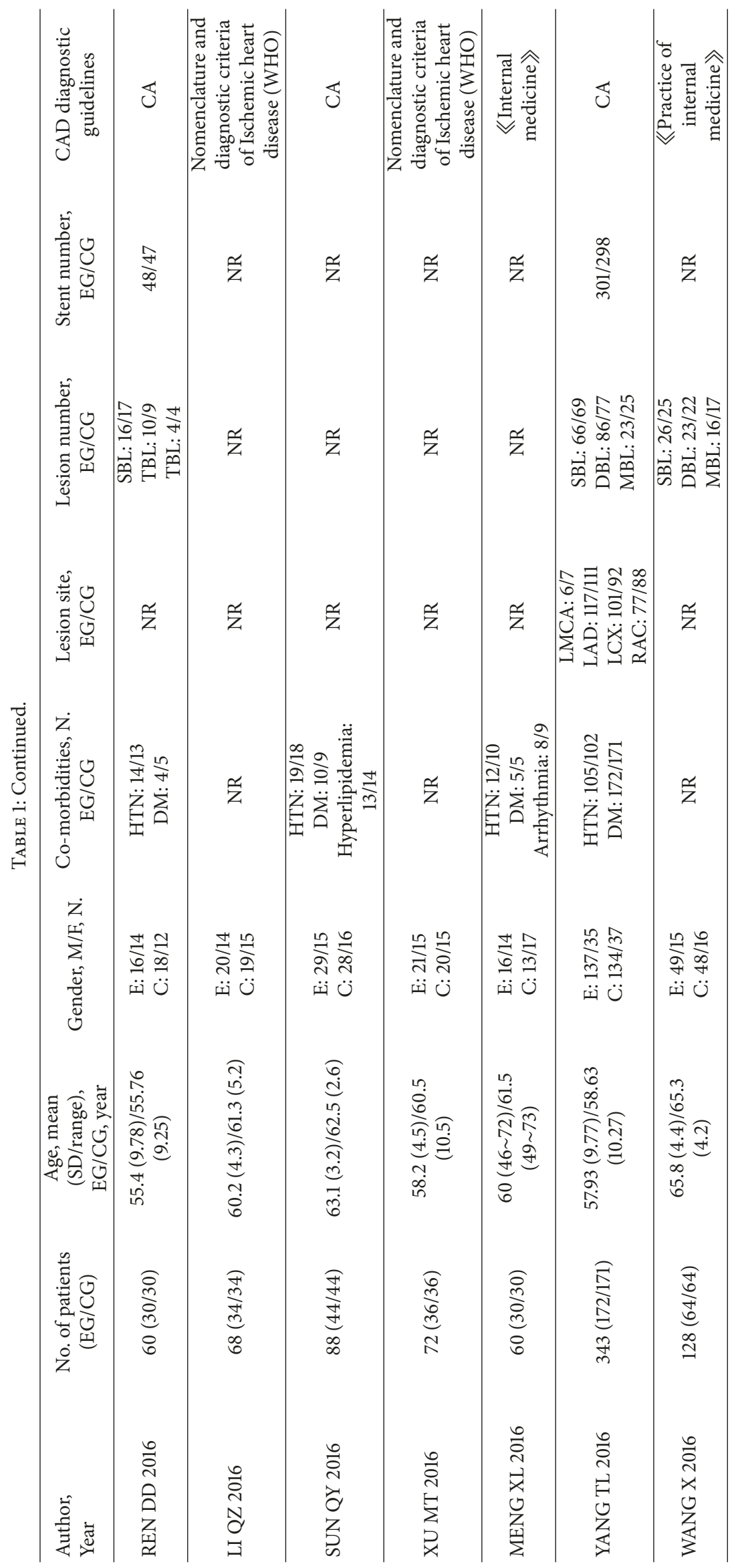




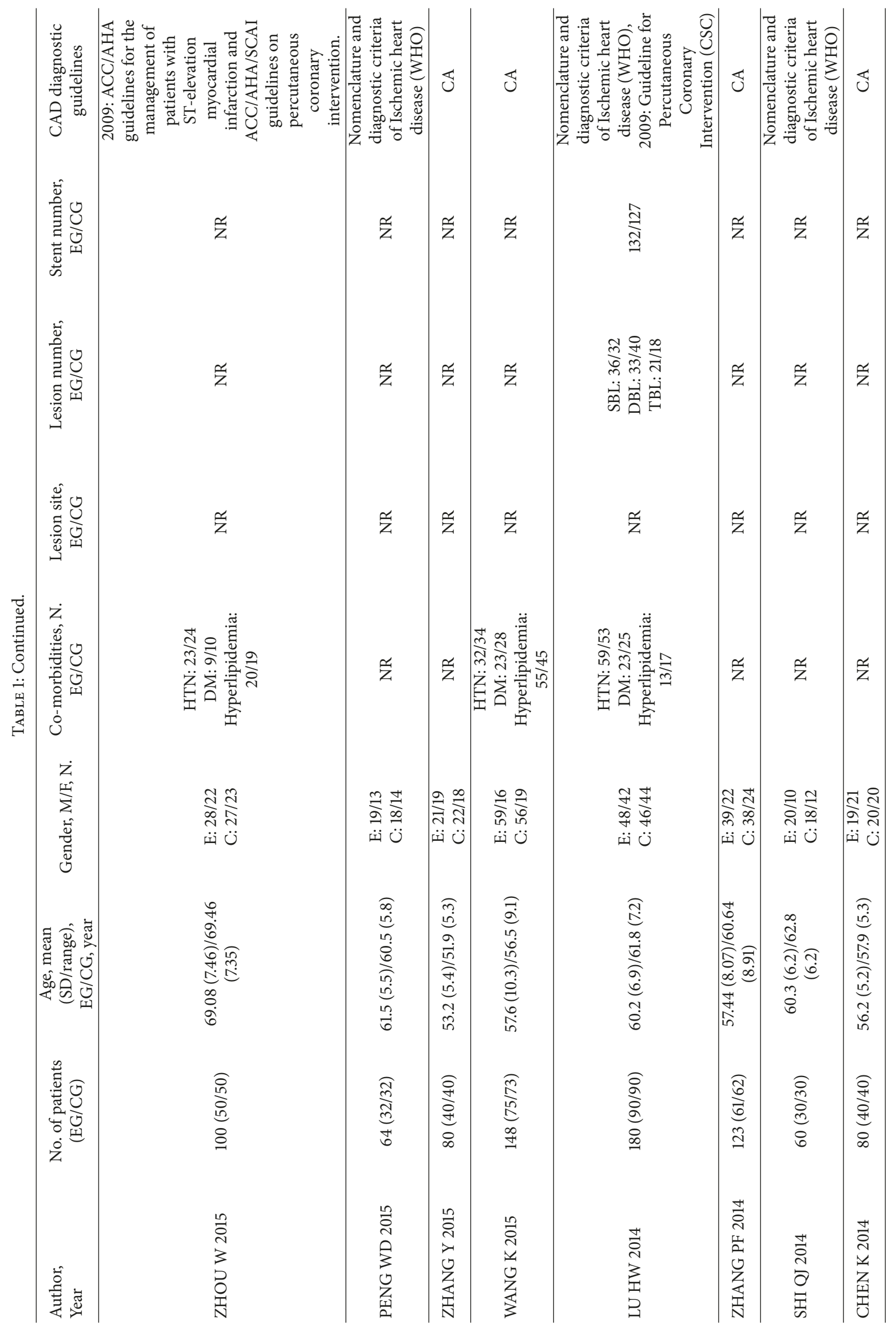




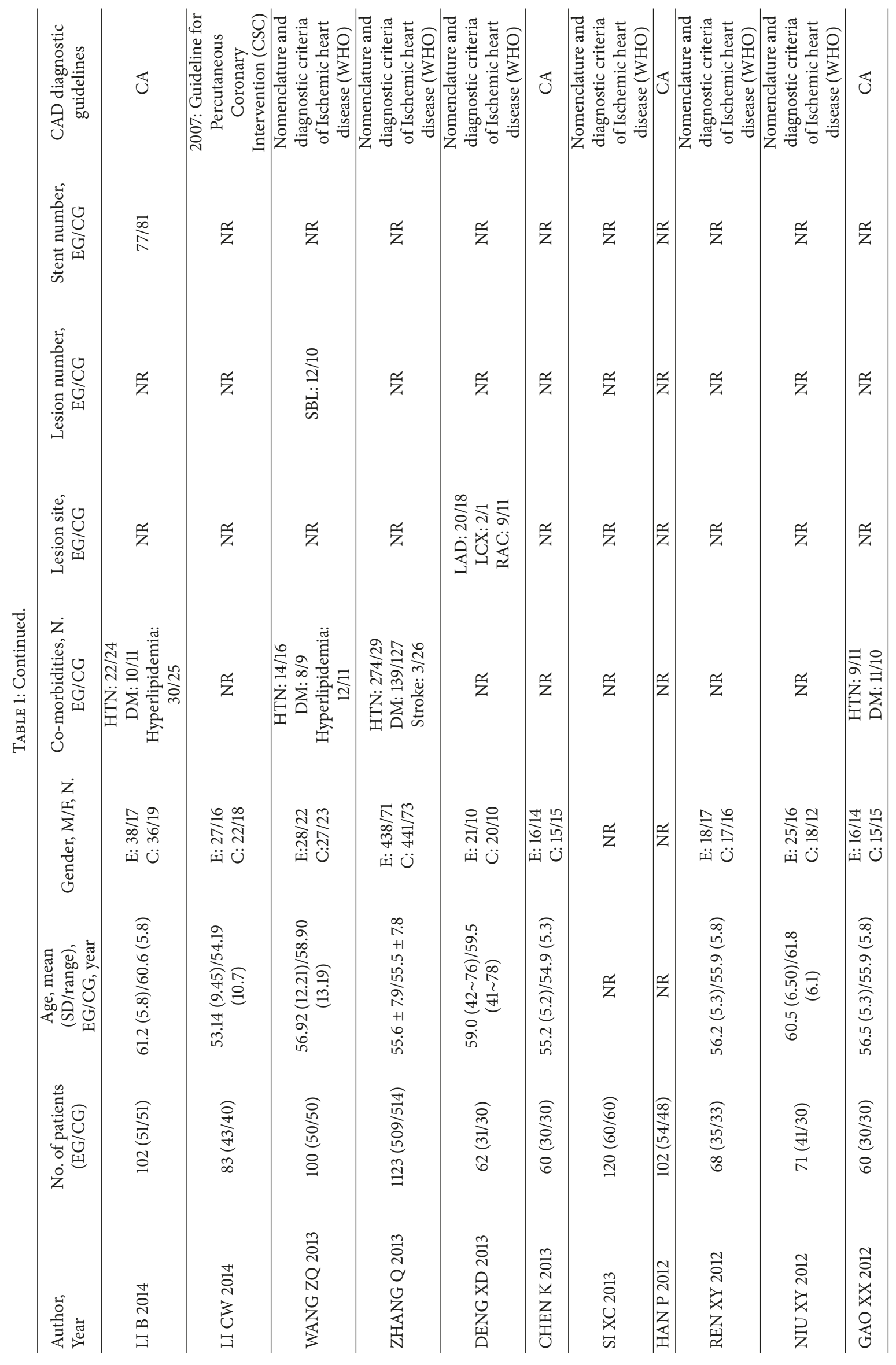




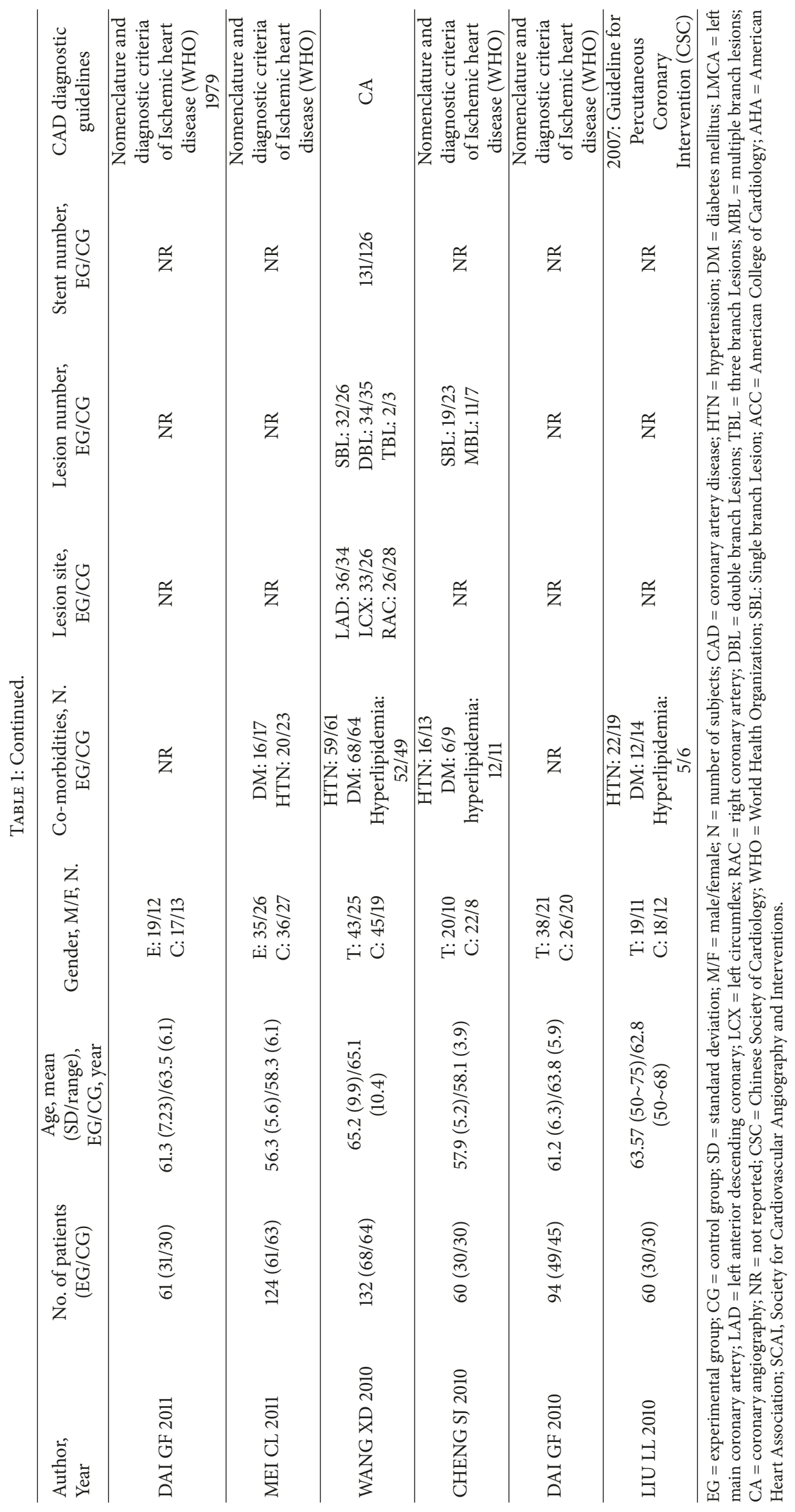




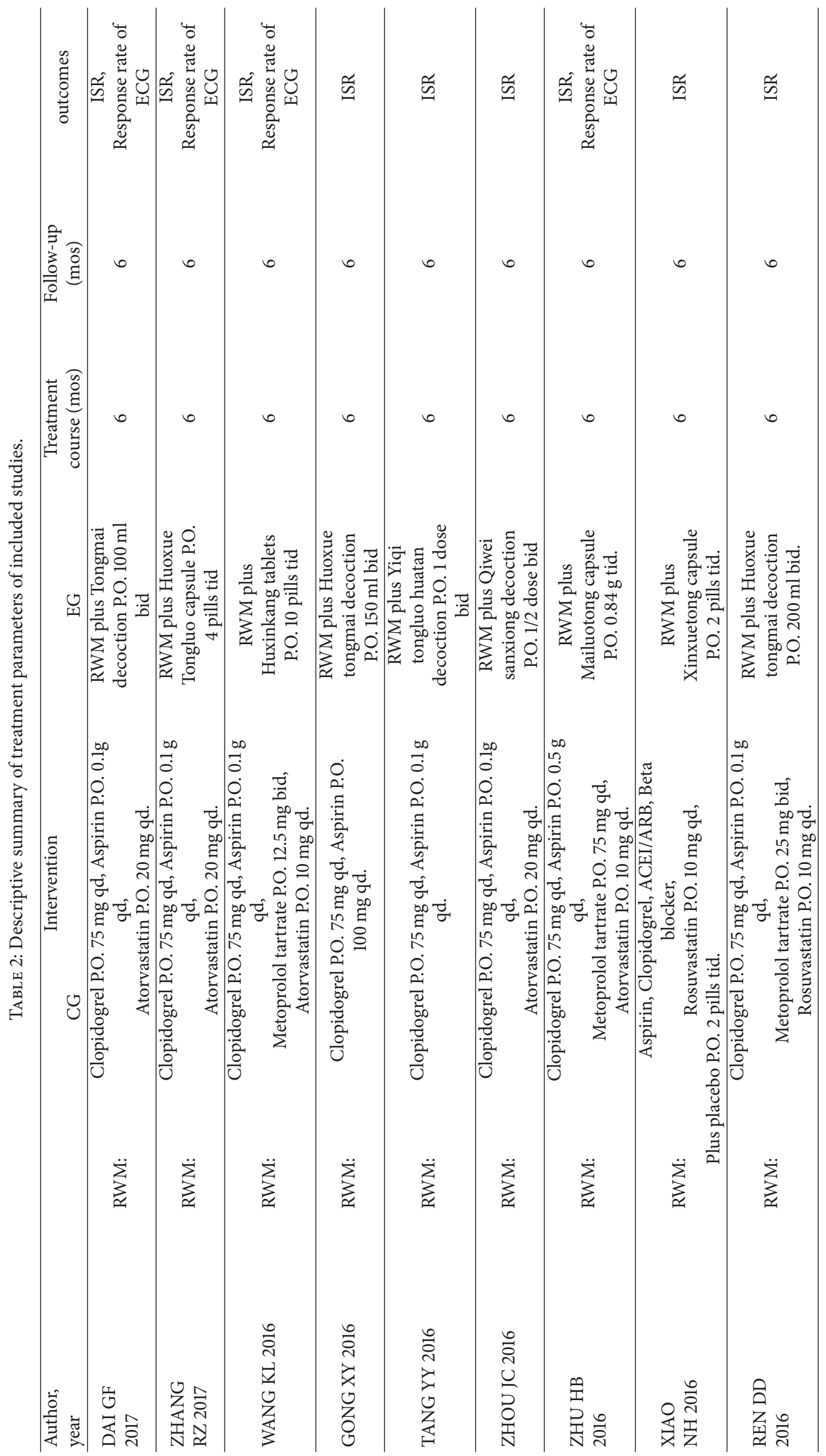




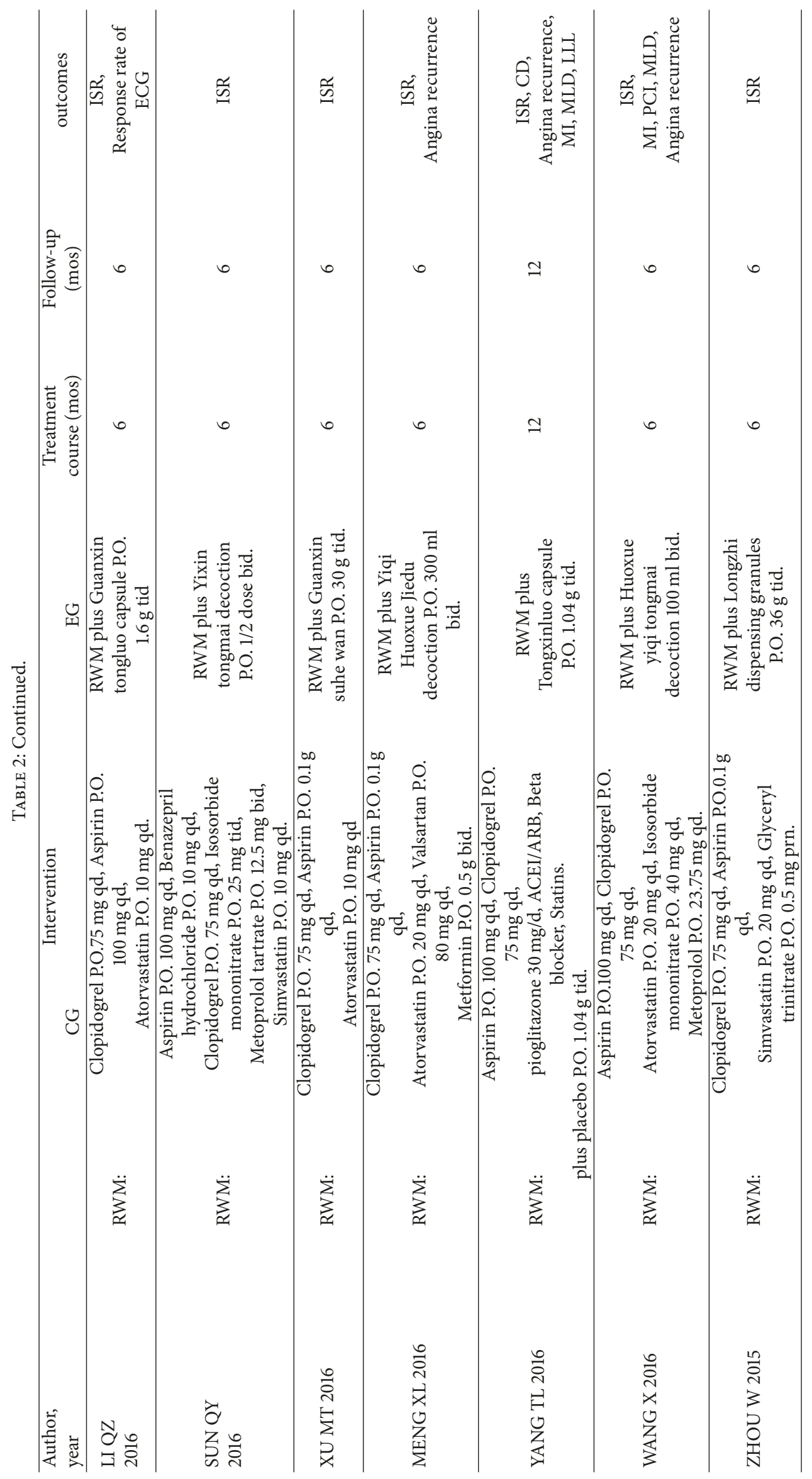




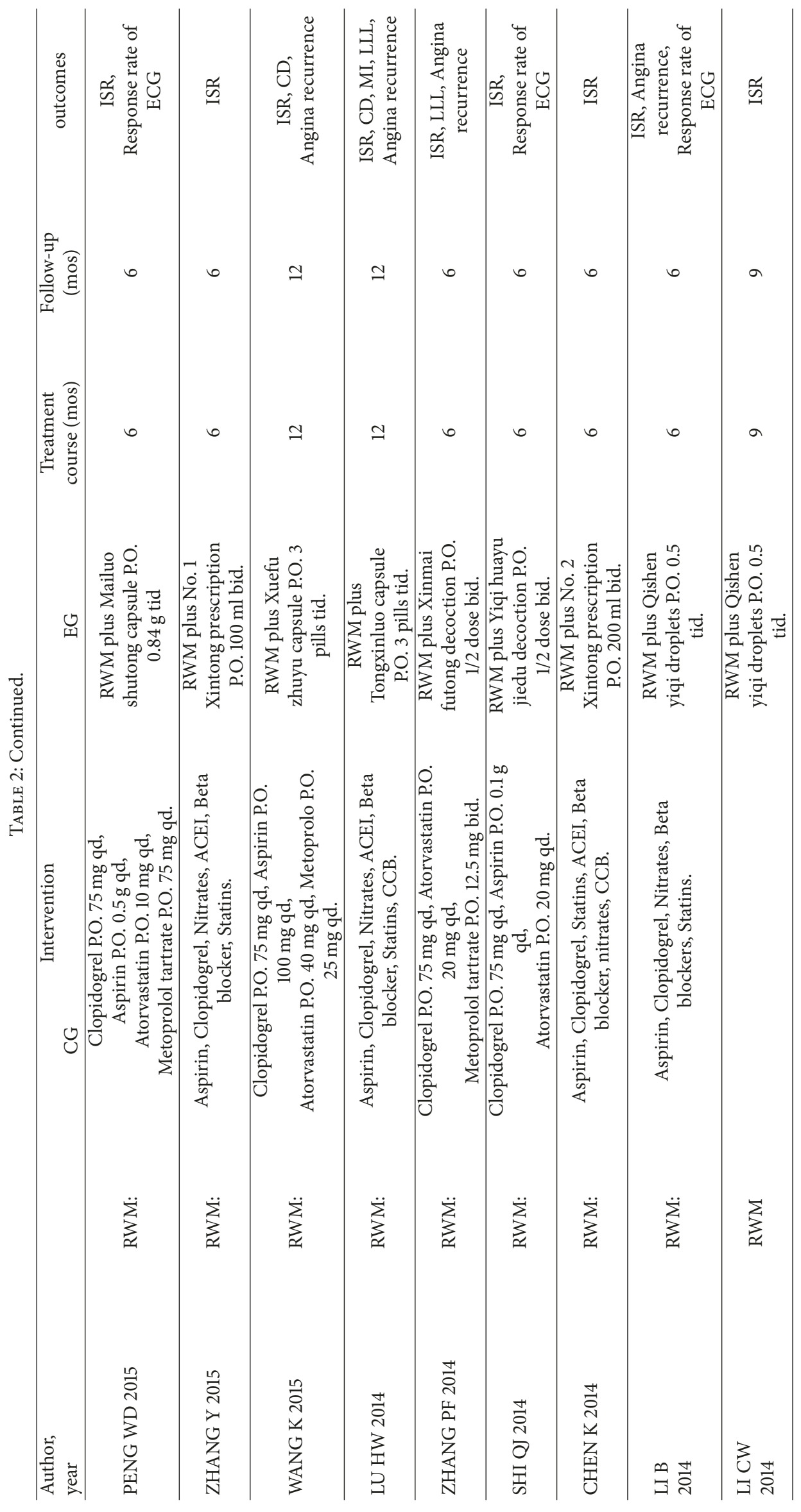




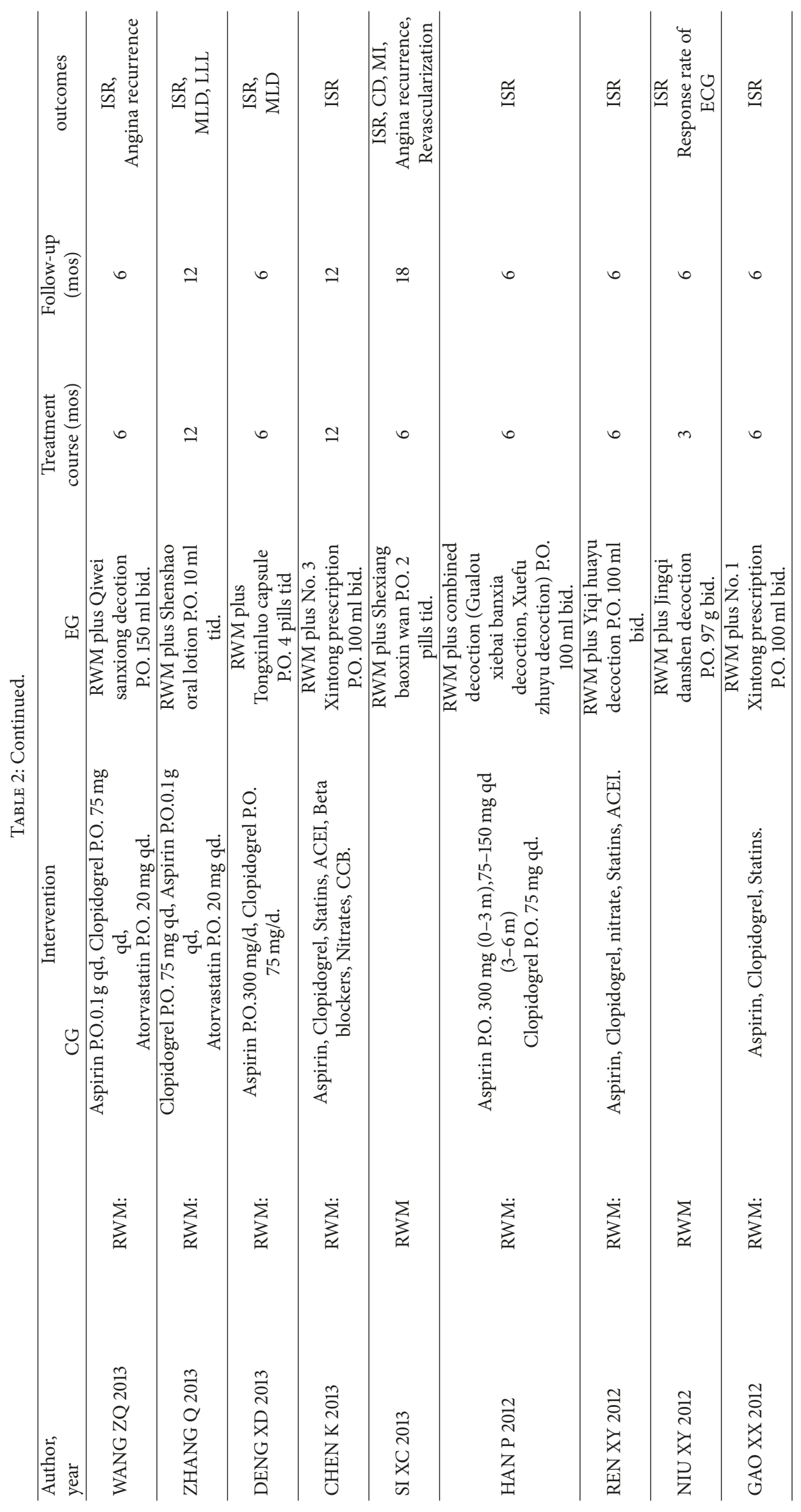




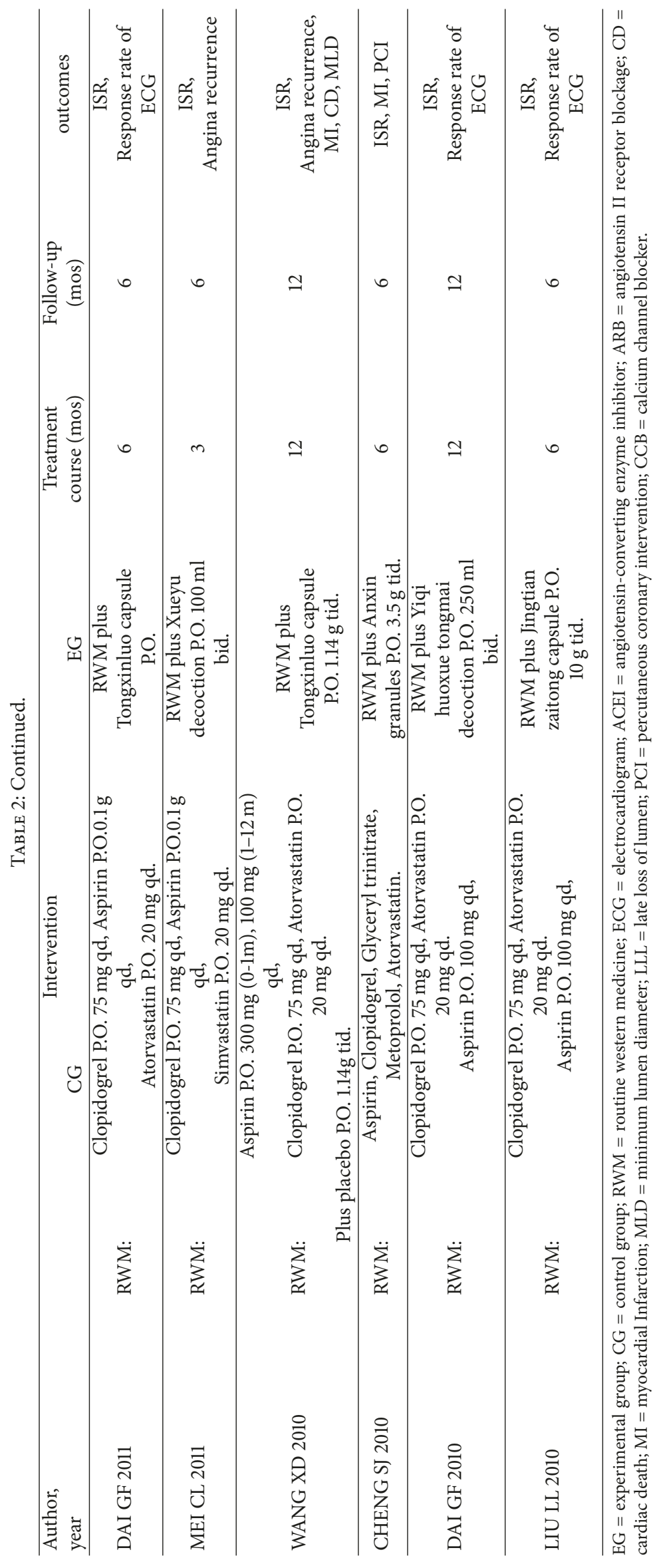


tables or SPSS software. The other 27 studies used the phrase "randomly allocating" but did not describe the method of randomization. Only 1 trial reported allocation concealment as "by sealed, opaque envelopes". Participants or outcome assessors were blinded in only 4 trials. All studies reported the primary outcome and clearly described the data collection methods, and we therefore believed that they were free from selective reporting bias. According to the criteria we prespecified, as shown in the Quality Assessment section, we judged the performance bias and detection bias of these studies to be low-risk. In conclusion, the overall methodological quality of the 40 trials was rated as low-risk. The details of this analysis are shown in Figure 2. The mean score for the RCTs included in this analysis was 5.15 (Table 3).

\subsection{Primary Outcomes}

3.4.1. In-Stent Restenosis. Three studies provided data for the number of ISR (NoR), and the pooled estimates showed that, in patients with CAD, NoR was lower for CCM plus RWM than for RWM alone (RR 0.24, 95\% CI 0.10 to $0.57, P=0.001$; $I^{2}=0 \%, P=0.81$ ) after 6 months of follow-up. In all, 34 studies provided data on the number of cases of ISR that had a follow-up time of 6 to 18 months. The rate of ISR was obviously lower in the CCM plus RWM group than in the group treated with the same RWM alone (RR 0.44, 95\% CI 0.37 to $\left.0.53, P<0.00001 ; I^{2}=0 \%, P=0.95\right)$. A metaanalysis of an additional 3 studies also showed that CCM plus RWM exerted a beneficial effect on the rate of ISR when CM placebo plus RWM was used as the control intervention with a 6- to 12 -month follow-up (RR $0.34,95 \%$ CI 0.20 to 0.57 , $\left.P<0.0001 ; I^{2}=0 \%, P=0.95\right)$. In addition, we evaluated these 34 studies to detect publication bias. The funnel plot was asymmetrical, indicating publication bias. Because funnel plot is used as a qualitative method to detect the publication bias of an article, we also used Egger's method to detect publication bias, and the results again implied the presence of publication bias $(P=0.00<0.05)$. The details of these analyses are shown in Figures 3-5 and Table 4.

3.4.2. Subgroup Analysis. Different types of Western medicine were used among the 34 studies, and we therefore performed a subgroup analysis. In 25 studies, a better benefit was exerted on ISR by CCM plus RWM (DAPT, DAPT + Statin, DAPT $+\beta$-blocker + Statin, Nitrates + DAPT $+\mathrm{ACEI} / \mathrm{ARB}+\beta$-blocker + Statin, Nitrates + DAPT + $\mathrm{ACEI} / \mathrm{ARB}+\beta$-blocker + Statin $+\mathrm{CCB})$ than the RWM alone. In 4 studies, there was no significant difference between the CCM plus RWM (Nitrates + DAPT + Statin, Nitrates + DAPT + ACEI/ARB + $\beta$-blocker + Statin) group and the RWM group (Table 5 and Figure 6 ).

Difference in the dosage forms of drugs (e.g., decoction, granules, pills, capsules, and tablets) might impact results, and we therefore carried out a subgroup analysis. A pooled estimate of cases of ISR showed that there was a significant difference between the decoction plus RWM and RWM alone groups in 18 studies ( $\mathrm{RR} 0.28,95 \% \mathrm{CI}=0.20$ to $0.38, P<$ $\left.0.00001 ; I^{2}=0 \%, P=0.97\right)$. No additional benefit was

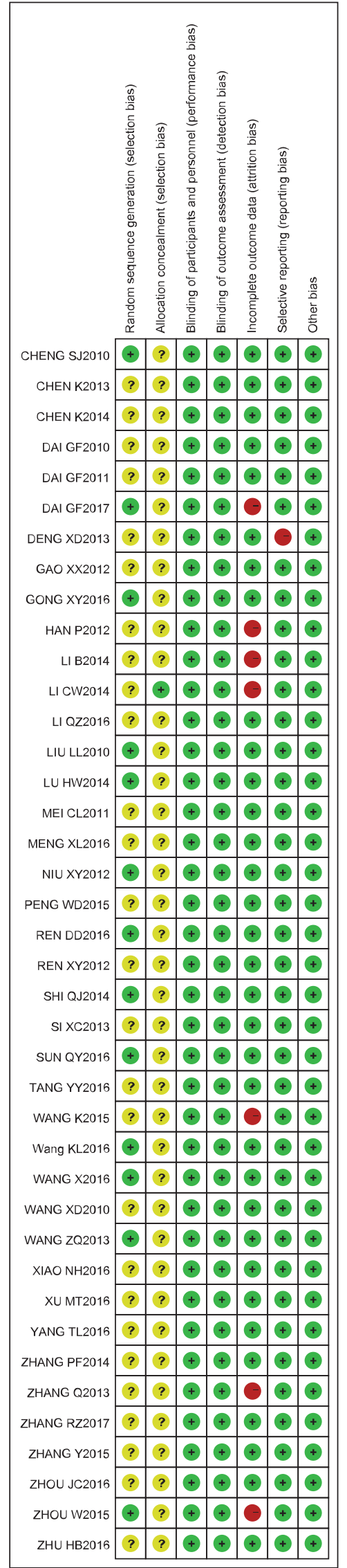

FIGURE 2: Summary of risk of bias in included studies. 


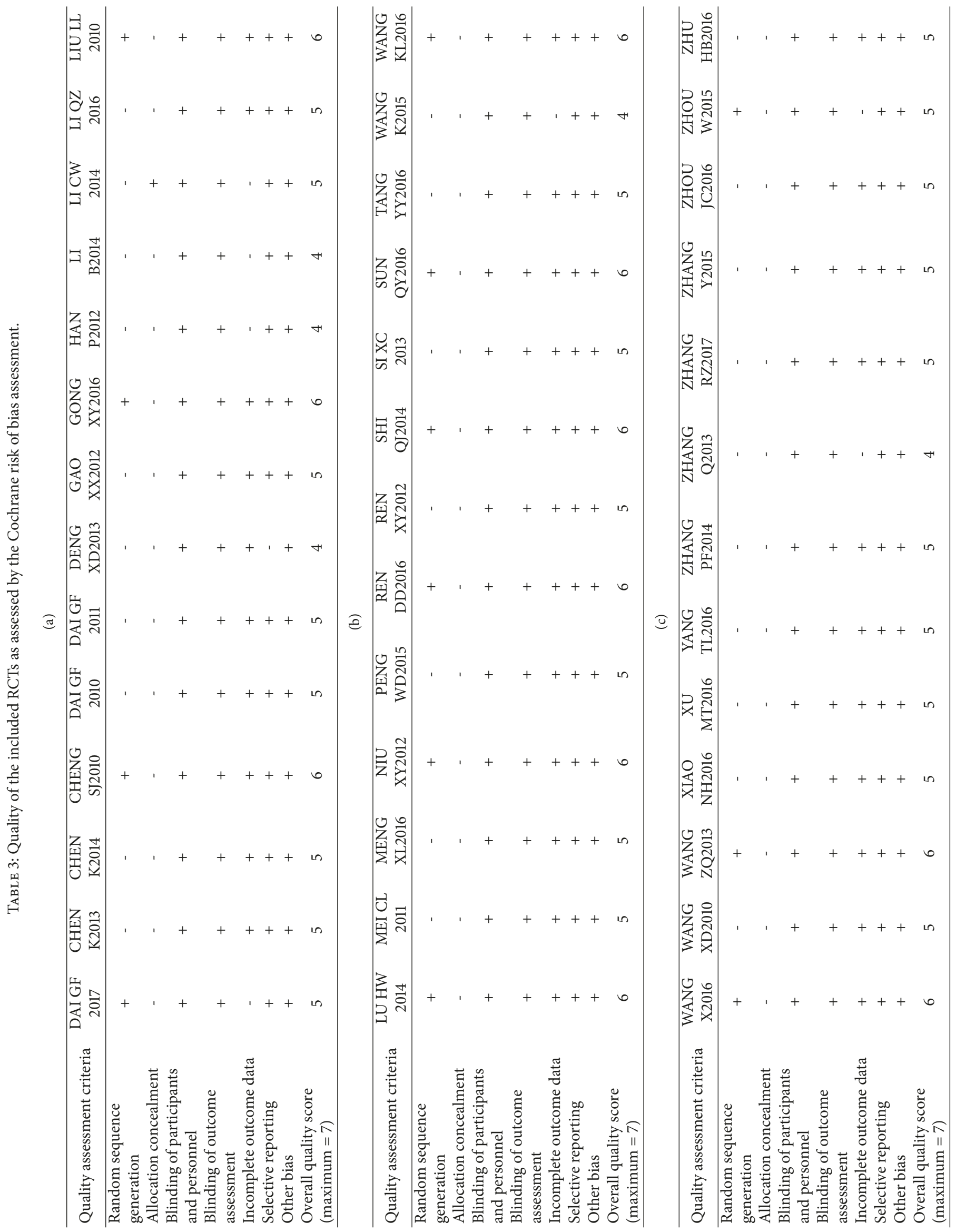




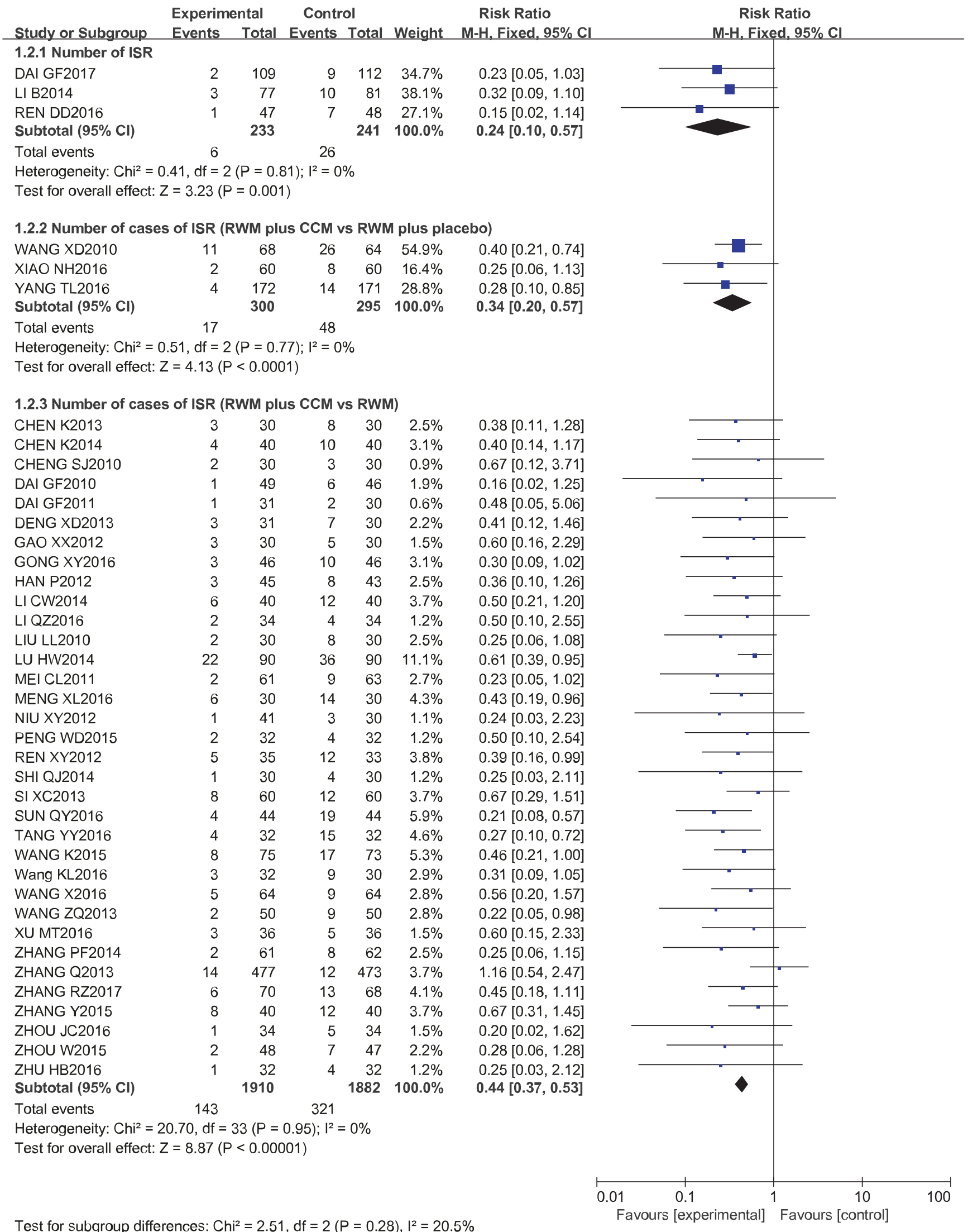

FIgURE 3: Meta-analysis of the effectiveness of CCM plus RWM in IRS. 
TABLE 4: Egger's test.

\begin{tabular}{lccccc}
\hline Std_Eff & Coef. & Std. Err. & $t$ & $P>|t|$ & [95\% Conf. Interval] \\
\hline slope & -.192833 & .1590705 & -1.21 & 0.234 & -0.5168491 \\
bias & -1.155985 & .3911951 & -3.97 & 0.000 & -1.74913 \\
\hline
\end{tabular}

TABLE 5: Subgroup analysis of CCM plus RWM versus RWM in cases of ISR.

\begin{tabular}{lccc}
\hline RWM & Studies $(n)$ & RR $(95 \%$ CI $)$ & $I^{2}$ \\
\hline DAPT & 4 & $0.32[0.18,0.57]$ & $0 \%$ \\
DAPT + Statin & 13 & $0.45[0.32,0.64]$ & $0.36[0.20,0.64]$ \\
DAPT + $\beta$-blocker + Statin & 4 & \\
DAPT + ACEI/ARB + Statin + Metformin & 1 & $0.36[0.12,1.08]$ \\
Nitrates + DAPT + Statin & 2 & $0.58[0.24,1.42]$ \\
Nitrates + DAPT + ACEI/ARB + Statin & 1 & $0.39[0.21,0.70]$ \\
Nitrates + DAPT + $\beta$-blocker + Statin & 2 & $0.57[0.37,0.86]$ \\
Nitrates + DAPT + ACEI/ARB + $\beta$-blocker + Statin & 2 & $0 \%$ \\
Nitrates + DAPT + ACEI/ARB + $\beta$-blocker + Statin + CCB & 2 & $0 \%$ \\
Unclear & 3 & $0 \%$ \\
\hline
\end{tabular}

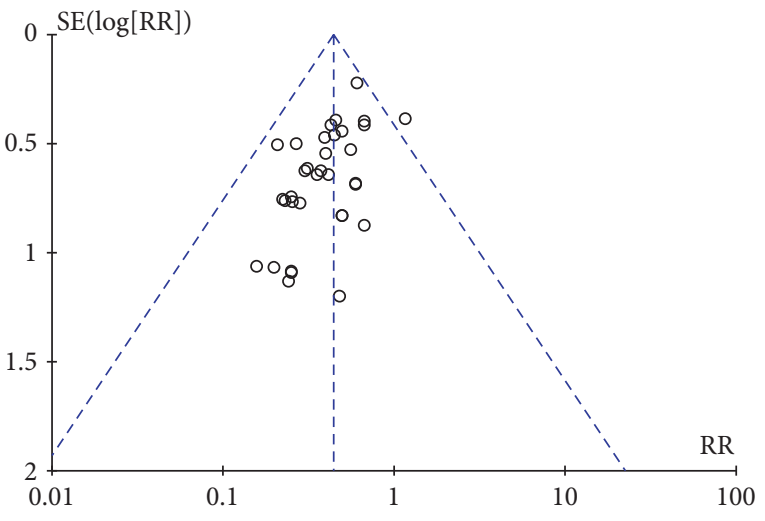

FIgURE 4: Funnel Plot of CCM plus RWM versus RWM.

exerted in ISR in the granules plus RWM group than in the RWM alone group (RR $0.39,95 \%$ CI 0.13 to $1.21, P=0.10$; $I^{2}=0 \%, P=0.46$ ). A meta-analysis of another 3 studies also showed that there was no significant difference between the pills plus RWM group and the RWM alone group (RR 0.59, $95 \% \mathrm{CI} 0.34$ to $1.01, P=0.60 ; I^{2}=0 \%, P=0.89$ ). There were significantly fewer cases of ISR in the capsules plus RWM group than in the group treated with the same RWM alone (RR $0.49,95 \%$ CI 0.36 to $0.67, P<0.00001 ; I^{2}=0 \%, P=$ 0.97). The details of these analyses are shown in Table 6 and Figure 7.

The association between the use of CCM plus RWM and RWM alone remained significant after the sensitivity analysis excluding studies with low quality score (quality score $\leqslant 4$ ) with a pooled RR of 0.41 (95\% CI, 0.34-0.50). (Figure 8).

3.4.3. Adverse Reactions. Only 2 studies reported the details of adverse reactions related to CCM, including hepatic dysfunction, gingival bleeding, diarrhoea, stomach discomfort,
TABLE 6: Subgroup analysis of CCM plus RWM versus RWM in cases of ISR.

\begin{tabular}{lccc}
\hline CCM & Studies $(n)$ & RR $(95 \%$ CI $)$ & $I^{2}$ \\
\hline decoction & 18 & $0.35[0.26,0.46]$ & $0 \%$ \\
granules & 2 & $0.39[0.13,1.21]$ & $0 \%$ \\
pills & 3 & $0.59[0.34,1.01]$ & $0 \%$ \\
capsules & 9 & $0.49[0.36,0.67]$ & $0 \%$ \\
tablets & 1 & & \\
\hline
\end{tabular}

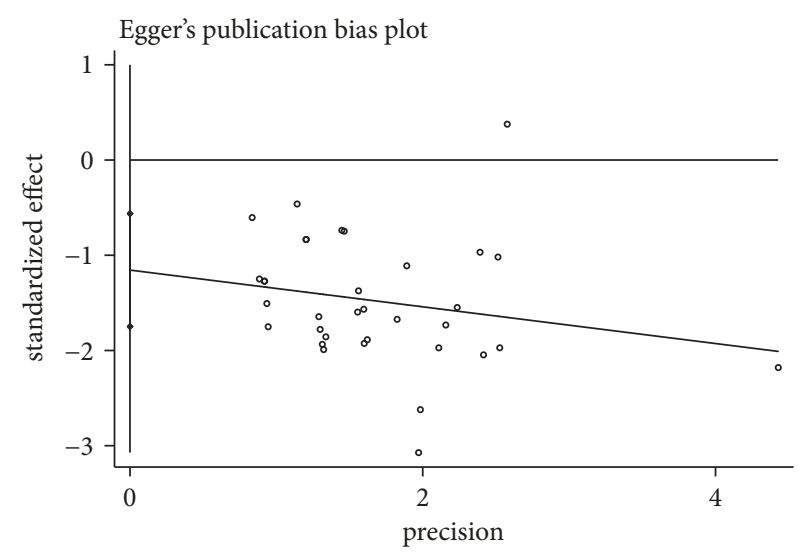

FIGURE 5: Egger's publication bias plot for CCM plus RWM vs RWM in cases of ISR.

flushing, dizziness, and headache. More adverse reactions were reported in patients treated with a Huoxue yiqi decoction and a Shenshao decoction, but most of these reactions were not severe and disappeared without special treatment. There was no significant difference in adverse reactions between the CCM plus RWM and RWM alone groups (Table 7 and Figure 9). 


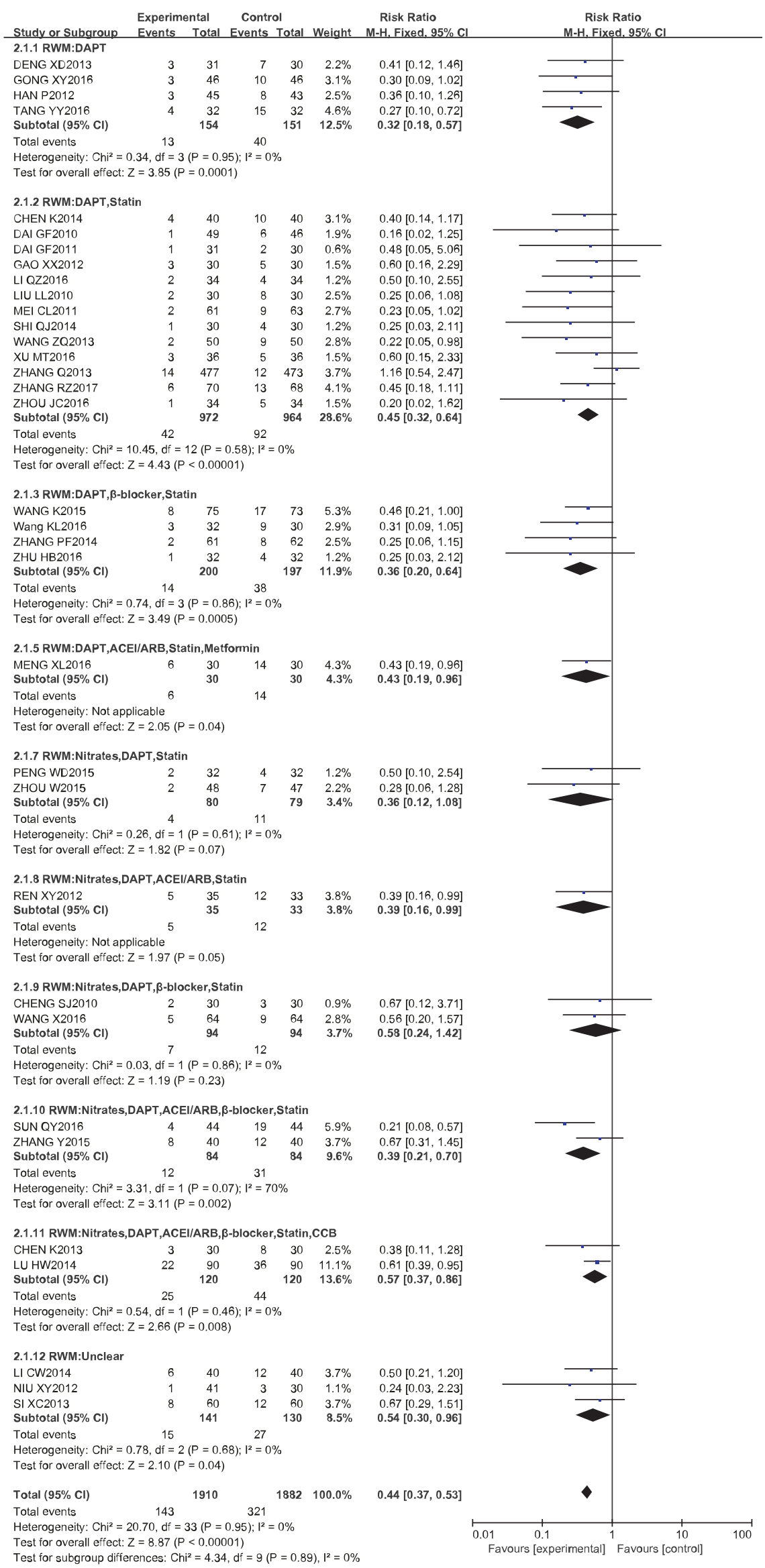

FIGURE 6: Meta-analysis of CCM plus RWM versus RWM in cases of ISR. 


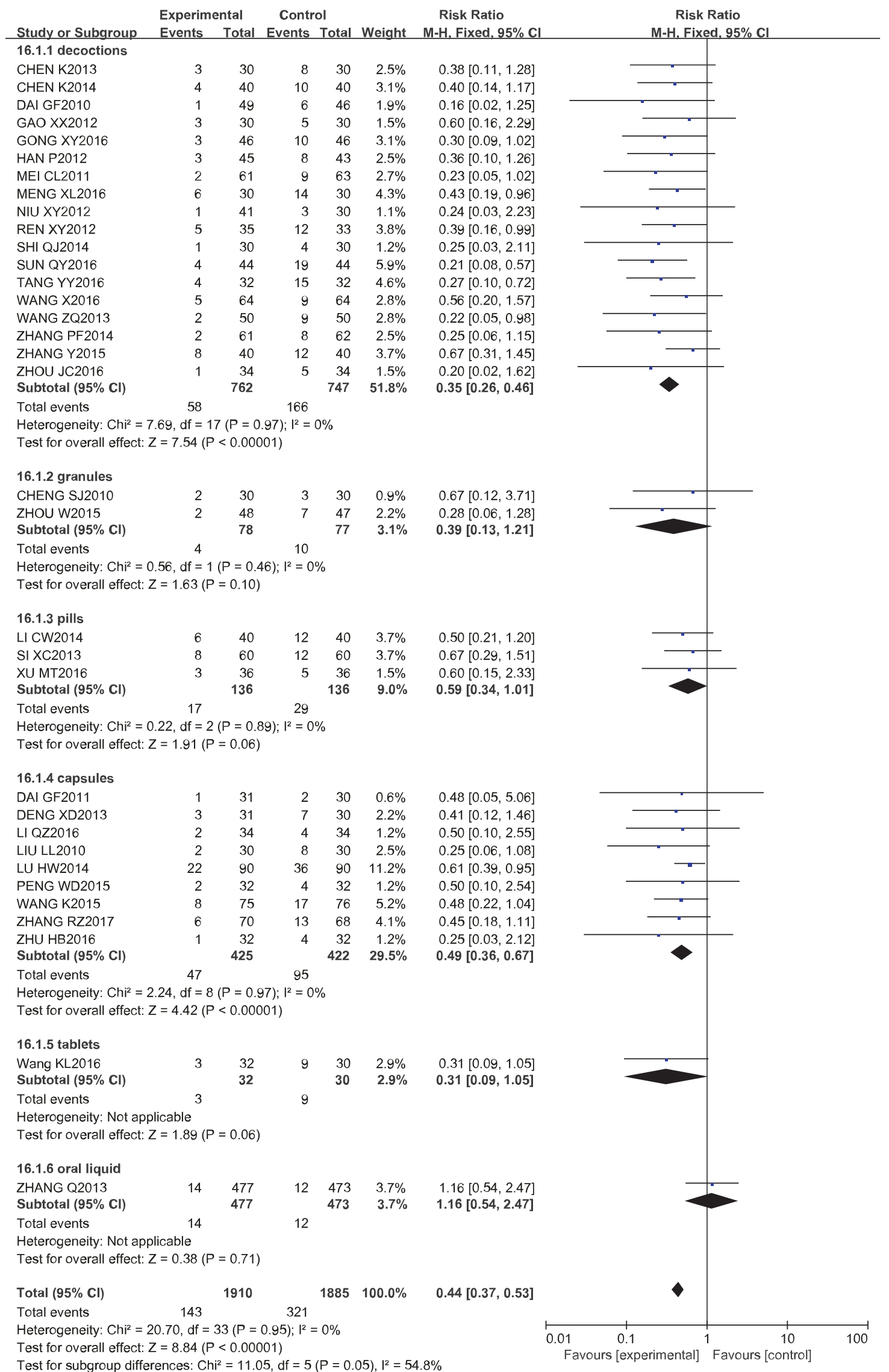

FIGURE 7: Meta-analysis of different CCM plus RWM versus RWM in cases of ISR. 
TABLE 7: Effect of CCM plus RWM versus RWM on Adverse Reactions.

\begin{tabular}{|c|c|c|c|c|c|c|}
\hline \multirow{2}{*}{ Study } & \multirow{2}{*}{ Intervention } & \multicolumn{2}{|c|}{ EG } & \multicolumn{2}{|c|}{ CG } & \multirow{2}{*}{ RR $[95 \% \mathrm{CI}]$} \\
\hline & & $\mathrm{EE}$ & Total & $\mathrm{EE}$ & Total & \\
\hline WANG X 2016 & $\begin{array}{l}\text { Huoxue yiqi decoction plus RWM versus } \\
\text { RWM }\end{array}$ & 8 & 64 & 5 & 64 & $1.60[0.55,4.63]$ \\
\hline ZHANG Q 2013 & $\begin{array}{c}\text { Shenshao Oral Lotion plus RWM versus } \\
\text { RWM }\end{array}$ & 2 & 473 & 1 & 477 & $2.02[0.18,22.17]$ \\
\hline
\end{tabular}

$\mathrm{EG}=$ experimental group; $\mathrm{CG}=$ control group; $\mathrm{EE}$ = events.

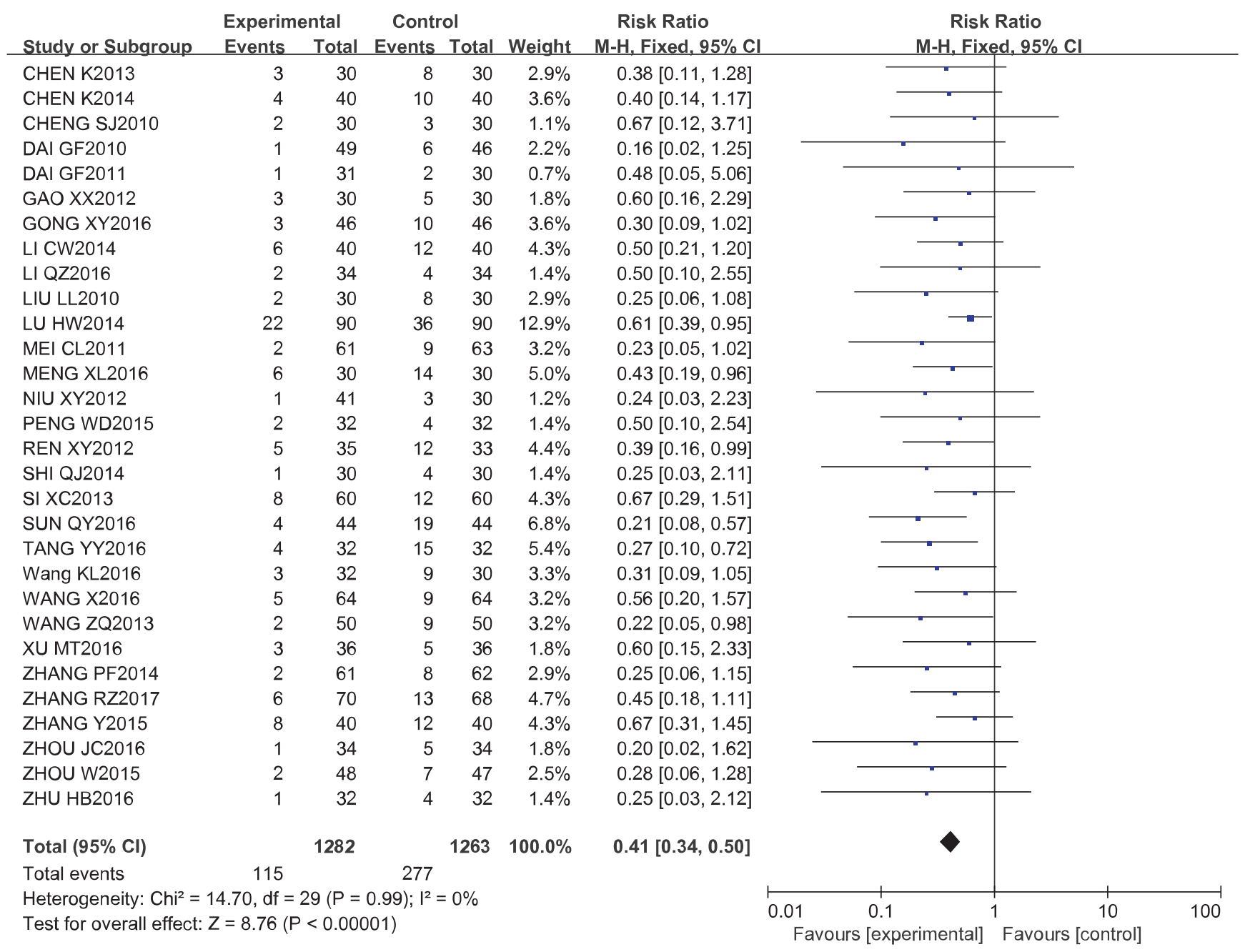

FIGURE 8: Sensitivity analysis excluding studies with low quality score of CCM plus RWM versus RWM in cases of ISR.

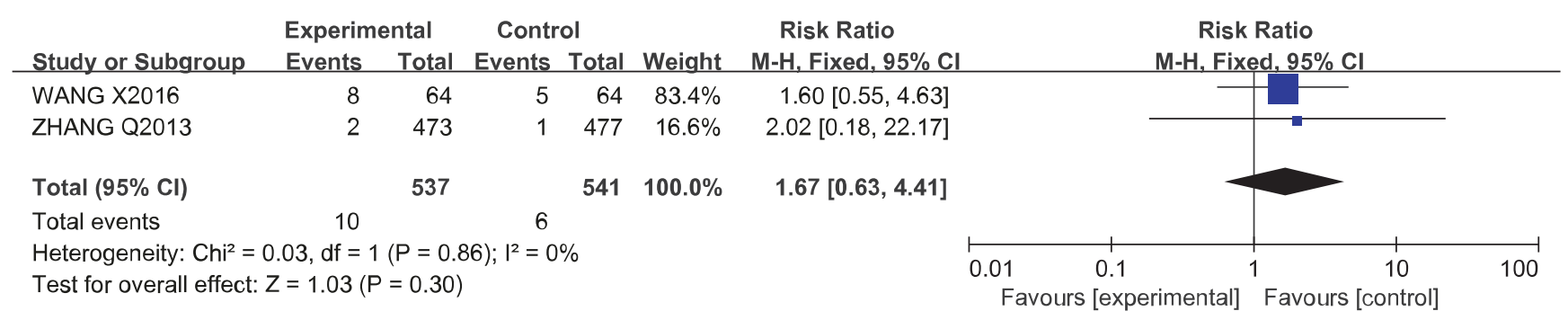

FIGURE 9: Meta-analysis of adverse reactions in the CCM plus RWM versus RWM groups. 
TABLE 8: Effect of CCM plus RWM versus RWM on MACE after PCI.

\begin{tabular}{lccc}
\hline MACE & No. of studies/patients & $\begin{array}{c}\text { Follow-up } \\
(\text { mos. })\end{array}$ & $\begin{array}{c}\text { Pooled estimate } \\
(\mathrm{RR}, 95 \% \mathrm{CI})\end{array}$ \\
\hline $\begin{array}{l}\text { Revascularization } \\
\text { (CCM plus RWM versus RWM) }\end{array}$ & $6-18$ & $0.33[0.07,1.62]$ \\
$\begin{array}{l}\text { Myocardial Infarction } \\
\text { (CCM plus RWM versus RWM) }\end{array}$ & $3 / 308$ & $6-18$ & $0.33[0.17,1.62]$ \\
$\begin{array}{l}\text { Myocardial Infarction } \\
\text { (CCM plus RWM versus CM placebo plus RWM) }\end{array}$ & $3 / 308$ & 6 & $0.42[0.11,1.60]$ \\
$\begin{array}{l}\text { Cardiac Mortality } \\
\text { (CCM plus RWM versus RWM) }\end{array}$ & $2 / 475$ & $12-18$ & $0.25[0.03,2.21]$ \\
$\begin{array}{l}\text { Recurrent Angina } \\
\text { (CCM plus RWM versus RWM) }\end{array}$ & $5 / 921$ & $6-18$ & $0.50[0.38,0.65]$ \\
$\begin{array}{l}\text { Recurrent Angina } \\
\text { (CCM plus RWM versus CM placebo plus RWM) }\end{array}$ & $9 / 1095$ & 6 & $0.32[0.19,0.54]$ \\
\hline
\end{tabular}

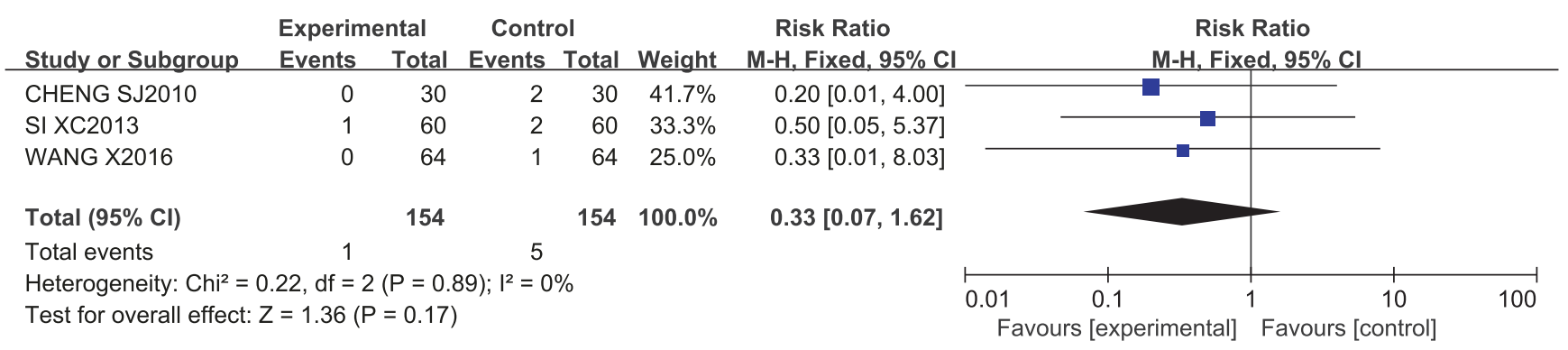

FIGURE 10: Meta-analysis of revascularization in CCM plus RWM versus RWM alone.

\begin{tabular}{|c|c|c|c|c|c|c|c|c|c|c|c|}
\hline Study or Subgroup & \multicolumn{2}{|c|}{ Experimental } & \multicolumn{2}{|c|}{ Control } & Weight & $\begin{array}{c}\text { Risk Ratio } \\
\text { M-H, Fixed, } 95 \% \mathrm{Cl}\end{array}$ & \multicolumn{3}{|c|}{$\begin{array}{c}\text { Risk Ratio } \\
\text { M-H, Fixed, } 95 \% \mathrm{Cl}\end{array}$} & & \\
\hline CHENG SJ2010 & 0 & 30 & 2 & 30 & $19.1 \%$ & $0.20[0.01,4.00]$ & & & & & \\
\hline SI XC2013 & 1 & 60 & 2 & 60 & $15.3 \%$ & $0.50[0.05,5.37]$ & & & & & \\
\hline WANG X2016 & 0 & 64 & 1 & 64 & $11.5 \%$ & $0.33[0.01,8.03]$ & & & & & \\
\hline WANG XD2010 & 1 & 68 & 2 & 64 & $15.8 \%$ & $0.47[0.04,5.06]$ & & & & & \\
\hline YANG TL2016 & 2 & 172 & 5 & 171 & $38.4 \%$ & $0.40[0.08,2.02]$ & & & & & \\
\hline Total $(95 \% \mathrm{Cl})$ & & 394 & & 389 & $100.0 \%$ & $0.38[0.14,1.05]$ & & & & & \\
\hline Total events & 4 & & 12 & & & & & & & & \\
\hline \multicolumn{7}{|c|}{ Heterogeneity: $\mathrm{Chi}^{2}=0.27, \mathrm{df}=4(\mathrm{P}=0.99) ; \mathrm{I}^{2}=0 \%$} & 0.0 & 0.1 & 1 & 10 & 100 \\
\hline \multicolumn{7}{|c|}{ Test for overall effect: $Z=1.86(P=0.06)$} & 0.4 & [experimental] & Favours $[\mathrm{c}$ & ontrol] & \\
\hline
\end{tabular}

FIGURE 11: Meta-analysis of myocardial infarction in CCM plus RWM versus RWM.

\subsection{Secondary Outcomes}

\subsubsection{Major Adverse Cardiac Events (Table 8)}

Revascularization. Revascularization was reported in 3 studies, in which 308 patients were treated with 3 different CCMs, including Anxin granules, Shexiang baoxin pills, and a Huoxue yiqi tongmai decoction. After 6-18 months of follow-up, the rate of revascularization was not significantly different between the CCM plus RWM and the RWM alone groups (RR $0.33,95 \%$ CI 0.07 to $1.62, P=0.17 ; I^{2}=0 \%$, $P=0.89$ ) (Figure 10).

Myocardial Infarction. Myocardial infarction (MI) was reported in 5 studies, in which 783 patients were treated with 4 different CCMs, including Anxin granules, Shexiang baoxin pills, Tongxinluo capsules, and a Huoxue yiqi tongmai decoction. During approximately 6-18 months of follow-up, no significant difference was found between the CCM plus RWM group and the RWM alone group (RR 0.33, 95\% CI 0.07 to $1.62, P=0.17 ; I^{2}=0 \%, P=0.89$ ). A meta-analysis of the other 2 studies also showed that there was no significant difference between the CCM plus RWM group and the CM placebo plus RWM group (RR $0.42,95 \%$ CI 0.11 to 1.60 , $\left.P=0.20 ; I^{2}=0 \%, P=0.91\right)$ after 6 months of follow-up (Figure 11).

Cardiac Mortality. In 5 studies of 4 kinds of CCM, 461 patients were included in the treatment group, and 460 patients were included in the control group. Cardiac death was recorded as 


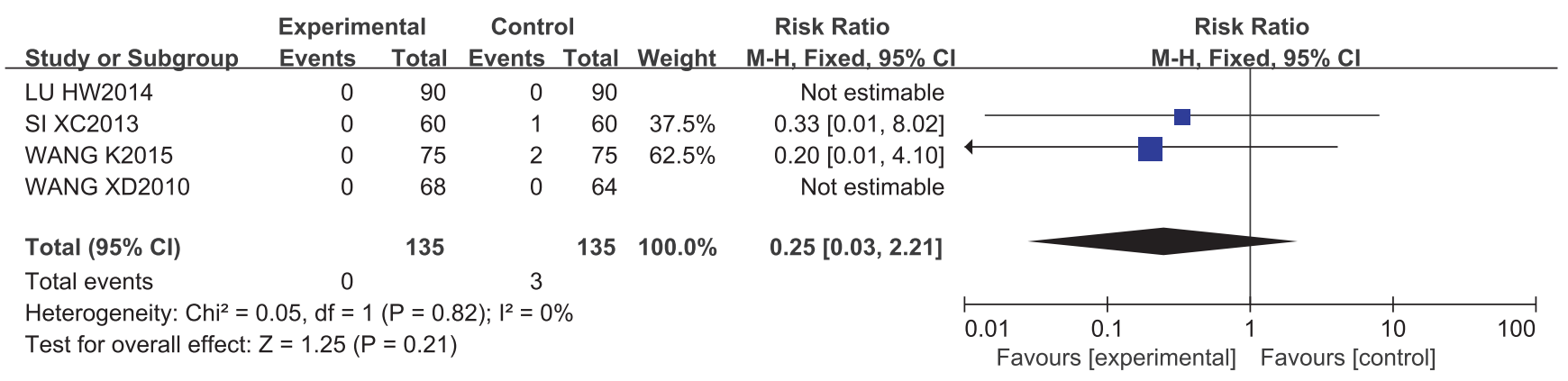

FIGURE 12: Meta-analysis of cardiac mortality in CCM plus RWM versus RWM.

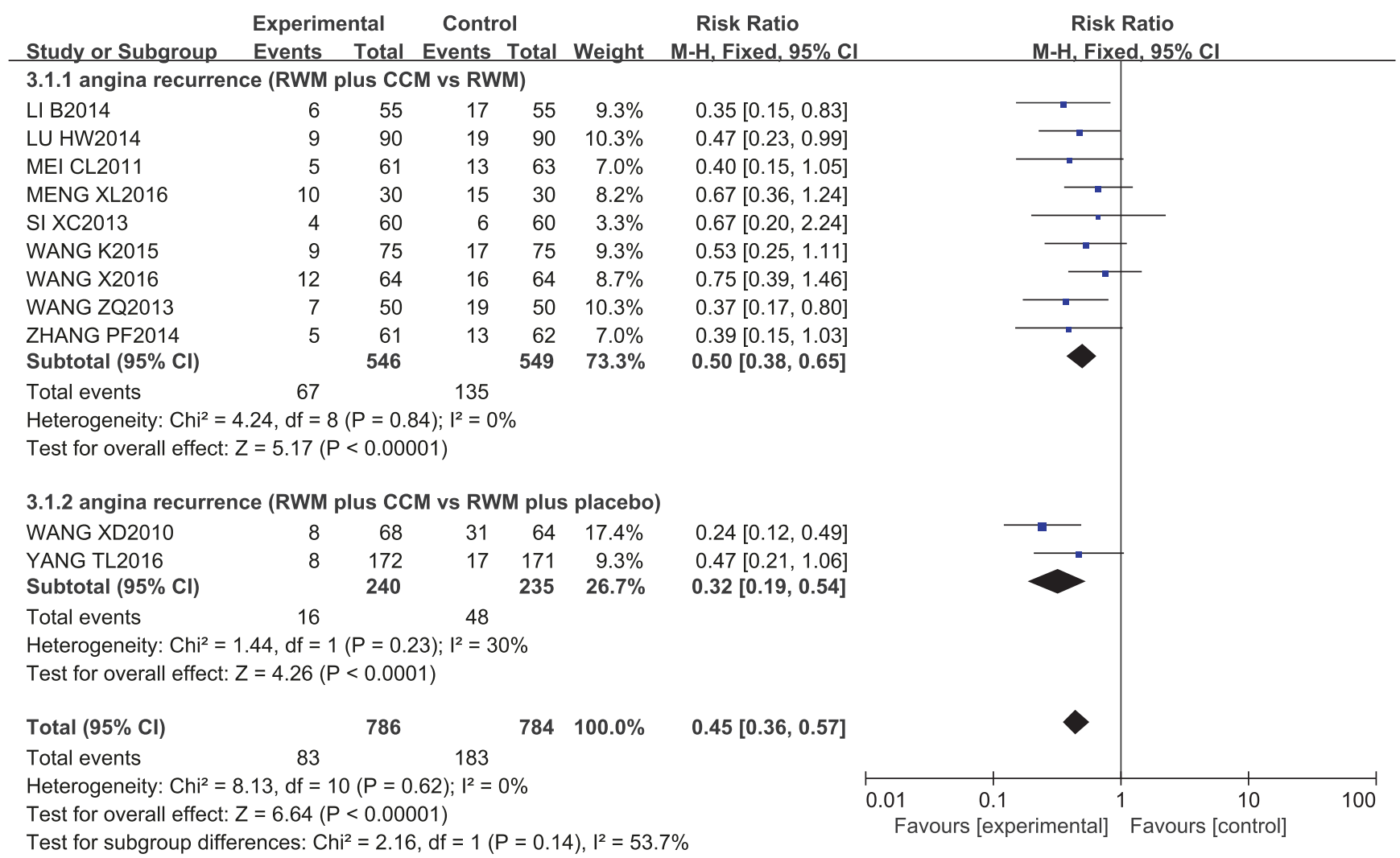

FIGURE 13: Meta-analysis of recurrent angina in CCM plus RWM versus RWM.

a measure of effect during a 12-18-month follow-up period. No cardiac deaths occurred in any of the groups in three of the studies or in the experimental group in the other 2 studies. A pooled estimate of cardiac deaths showed that there was no significant difference between the CCM plus RWM and RWM alone groups in the latter two studies (RR 0.25, 95\% CI $=0.03$ to $2.21 ; P=0.21 ; I^{2}=0 \%, P=0.82$ ) (Figure 12).

Recurrent Angina. Recurrent angina was reported in 11 studies involving 1570 patients. During a 6-18-month follow-up period, the rate of recurrent angina was significantly lower in the CCM plus RWM group than in the groups treated with the same RWM alone (RR $0.50,95 \%$ CI 0.38 to $0.65, P<0.00001$; $I^{2}=0 \%, P=0.84$ ) or with a CM placebo plus the same RWM
(RR 0.32, 95\% CI 0.19 to $0.54, P<0.00001 ; I^{2}=30 \%, P=$ 0.23) (Figure 13).

Angiographic Measurements. Follow-up angiography performed in patients with ISR at 6-12 months after the index PCI in 7 studies that evaluated 4 Chinese Herbal Medicines (CHMs) in 1917 patients. Baseline information showed that the mean minimal luminal diameter (MLD) before and immediately after the index PCI and the gain in luminal diameter following stent placement were comparable between groups.

MLD is defined as the smallest diameter in the treated lesion area [53] and was reported in 5 studies (Table 9). MLD was significantly better in the CCM plus RWM group than 
TABLE 9: Effect of CCM on MLD.

\begin{tabular}{|c|c|c|c|c|c|c|c|c|}
\hline \multirow{2}{*}{ Study } & \multirow{2}{*}{ Intervention } & \multicolumn{3}{|c|}{ EG } & \multicolumn{3}{|c|}{ CG } & \multirow{2}{*}{$\mathrm{MD}[95 \% \mathrm{CI}]$} \\
\hline & & Mean & $\mathrm{SD}$ & Total & Mean & $\mathrm{SD}$ & Total & \\
\hline DENG XD 2013 & $\begin{array}{l}\text { Tongxinluo capsule plus } \\
\text { RWM versus RWM }\end{array}$ & 3.97 & 0.59 & 31 & 5.58 & 0.67 & 30 & $-1.61[-1.93,1.29]$ \\
\hline WANG X 2016 & $\begin{array}{l}\text { Huoxue yiqi tongmai } \\
\text { decoction plus RWM vs } \\
\text { RWM }\end{array}$ & 2.02 & 0.22 & 64 & 1.6 & 0.2 & 64 & $0.42[0.35,0.49]$ \\
\hline WANG XD 2010 & $\begin{array}{c}\text { Tongxinluo capsule plus } \\
\text { RWM versus CM placebo } \\
\text { plus RWM }\end{array}$ & 2.43 & 0.62 & 68 & 1.16 & 0.87 & 64 & $1.27[1.01,1.53]$ \\
\hline YANGN TL 2016 & $\begin{array}{c}\text { Tongxinluo capsule plus } \\
\text { RWM versus CM placebo } \\
\text { plus RWM }\end{array}$ & 2.81 & 0.41 & 172 & 2.46 & 0.37 & 171 & $0.35[0.27,0.43]$ \\
\hline ZHANG Q 2013 & $\begin{array}{c}\text { Shenshao oral lotion plus } \\
\text { RWM versus RWM }\end{array}$ & 2.11 & 0.38 & 473 & 2.09 & 0.32 & 477 & $0.02[-0.02,0.06]$ \\
\hline
\end{tabular}

$\mathrm{EG}=$ experimental group; $\mathrm{CG}=$ control group; $\mathrm{MD}=$ mean difference.

TABLE 10: Effect of CCM on LLL.

\begin{tabular}{|c|c|c|c|c|c|c|c|c|}
\hline \multirow{2}{*}{ Study } & \multirow{2}{*}{ Intervention } & \multicolumn{3}{|c|}{ EG } & \multicolumn{3}{|c|}{ CG } & \multirow{2}{*}{$\mathrm{MD}[95 \% \mathrm{CI}]$} \\
\hline & & Mean & SD & Total & Mean & SD & Total & \\
\hline $\begin{array}{l}\text { LU HW } \\
2014\end{array}$ & $\begin{array}{l}\text { Tongxinluo capsule plus } \\
\text { RWM versus RWM }\end{array}$ & 0.43 & 0.17 & 90 & 0.79 & 0.24 & 90 & $-0.36[-0.42,0.30$ \\
\hline YANGN TL 2016 & $\begin{array}{c}\text { Tongxinluo capsule plus } \\
\text { RWM versus CM placebo } \\
\text { plus RWM }\end{array}$ & 0.21 & 0.17 & 172 & 0.45 & 0.24 & 171 & $-0.24[-0.28,0.20]$ \\
\hline ZHANG PF 2014 & $\begin{array}{l}\text { Xinmai futong decoction } \\
\text { plus RWM versus RWM }\end{array}$ & 0.5 & 0.21 & 61 & 0.8 & 0.4 & 62 & $-0.30[-0.41,0.19]$ \\
\hline ZHANG Q 2013 & $\begin{array}{l}\text { Shenshao oral lotion plus } \\
\text { RWM versus RWM }\end{array}$ & 0.62 & 0.3 & 473 & 0.67 & 0.32 & 477 & $-0.05[-0.09,0.01]$ \\
\hline
\end{tabular}

$\mathrm{EG}=$ experimental group; $\mathrm{CG}=$ control group; $\mathrm{MD}=$ mean difference.

in the CM placebo plus RWM when patients were treated with Tongxinluo capsules (MD WANG XD2010 $1.27 \mathrm{~mm}, 95 \% \mathrm{CI}$ 1.01 to $1.53 \mathrm{~mm} ; \mathrm{MD}_{\text {YANG TL2016 }}=0.35 \mathrm{~mm}$, 95\% CI 0.27 to $0.43 \mathrm{~mm}$ ) and in the CCM plus RWM group than in the group treated with the same RWM alone when a Huoxue yiqi tongmai decoction was evaluated ( $\mathrm{MD}_{\text {WANG X2016 }} 0.42 \mathrm{~mm}$, $95 \%$ CI 0.35 to $0.49 \mathrm{~mm}$ ). There was no significant difference between the groups in the studies evaluating Shenshao oral lotion $\left(\mathrm{MD}_{\text {ZHANG Q2016 }}=0.02 \mathrm{~mm}, 95 \% \mathrm{CI}-0.02\right.$ to $\left.0.06 \mathrm{~mm}\right)$. The Tongxinluo capsule plus RWM did not improve MLD when patients were compared to those treated with the same RWM alone $\left(\mathrm{MD}_{\mathrm{DENG} X \mathrm{XD} 2013}=-1.61 \mathrm{~mm}, 95 \% \mathrm{CI}-1.93\right.$ to $-1.29 \mathrm{~mm})$.

The primary angiographic endpoint was late lumen loss (LLL) after 6 to 12 months. LLL was evaluated by determining the difference between the minimum lumen diameter after the procedure and at follow-up using quantitative coronary angiography [54]. LLL was measured in 4 studies of 3 CHMs after 6 and 12 months of follow-up (Table 10). There was a significant difference between the CHM plus RWM group and the control group treated with the same RWM alone when the CHM being tested was Tongxinluo capsules $\left(\mathrm{MD}_{\mathrm{LU} \mathrm{HW} 2014}=-0.36 \mathrm{~mm}, 95 \% \mathrm{CI}-0.42\right.$ to $\left.-0.30 \mathrm{~mm}\right)$, a Xinmai futong decoction ( $\mathrm{MD}_{\mathrm{ZHANG} \text { PF2014 }}=-0.3 \mathrm{~mm}$,
95\% CI -0.41 to $-0.19 \mathrm{~mm}$ ) and a Shenshao oral lotion $\left(\mathrm{MD}_{\text {ZHANG Q2013 }}=-0.05 \mathrm{~mm}, 95 \% \mathrm{CI}-0.09\right.$ to $\left.-0.01 \mathrm{~mm}\right)$. Finally, LLL was lower in the RWM plus Tongxinluo capsule group than in the group treated with the same RWM plus placebo $\left(\mathrm{MD}_{\text {YANGN TL2016 }}=-0.24 \mathrm{~mm}, 95 \% \mathrm{CI}-0.28\right.$ to $-0.20 \mathrm{~mm})$.

In this SR, 40 studies involving $4536 \mathrm{CAD}$ patients who underwent stent implantation were identified. All studies were RCTs. Approximately $75 \%$ of the studies were performed in the last five years. A domain-based evaluation showed that the mean score of the included RCTs was 5.15. Of the included studies, three were designed to compare a CCM plus RWM versus a CM placebo plus the same RWM, and 37 studies were designed to compare a CCM plus RWM versus the same RWM alone.

In this SR, all studies assessed ISR using CTA or CA. A three subgroup meta-analysis of 33 CCM plus RWM groups showed that they produced an absolute decrease in the ISR rate. Given the low risk of bias (demonstrated by analyses of methodological quality and publication bias), the following robust conclusion can be drawn: CCM plus RWM reduces ISR. Furthermore, there was no significant difference in adverse reactions between the CCM plus RWM and RWM alone groups in 2 of the studies, potentially indicating 
that CCM plus RWM is a safe treatment. However, further observations are needed. An Egger's linear regression test was performed to statistically assess funnel plot asymmetry and publication bias. Substantially more studies in the literature have positive than negative results, and this produced socalled publication bias.

With regard to secondary outcomes, we concluded that CCM plus RWM did not exert a benefit against revascularization, MI and cardiac mortality during a 6-18-month follow-up period after stent implantation. This finding may be attributed to the limited number of patients and the short follow-up times reported in the studies. However, 11 studies explored CCMs plus RWMs, and recurrent angina was significantly reduced by this treatment during the 6-18month follow-up period. This may indicate that treatment with a CCM plus RWM exerts a better effect on symptom improvement. Some studies have concluded that a major determinant of the restenosis rate is the final MLD after all interventions [55]. MLD can be evaluated as a continuous end point from 6 to 9 months after intervention and is precisely associated with stable long-term results in the treated coronary segment [56]. In addition, LLL is monotonically correlated with the probability of restenosis and, when evaluated, is a more efficient estimate of restenosis progress in this era of lower binary restenosis rates [54]. Hence, MLD and LLL are two major determinants of the restenosis rate. In this SR, 7 studies that evaluated 4 CHMs reported MLD and LLL. Although pooled results were not available for either MLD or LLL because there was high heterogeneity $\left(I^{2}=97 \%\right.$ or $I^{2}=99 \%$ ), in most of the studies, CCM plus RWM exerted significant beneficial effects.

Specifically, Tongxinluo capsules appeared to markedly reduce the rates of ISR, recurrent angina, and LLL and improve MLD. Qiwei sanxiong decoction [37] appeared to markedly reduce the rates of ISR and recurrent angina. Yiqi huoxue huayu decoction [24], Yiqi huayu decoction [43], Yixin tongmai decoction [22], and Yiqi tongluo huatan decoction [16] each provided significant benefits by reducing the rate of ISR. Qishen yiqi droplets [35] exerted significant benefits by reducing recurrent angina. Most of the abovedescribed CCMs are meant to supplement Qi and activate the blood. Their main ingredients are Huangqi (astragalus), Renshen (Panax), Shaoyao (peony), Danshen (salvia), and Chuanxiong (tetramethylpyrazine).

The pathogenesis of restenosis is not yet fully understood. However, it is generally accepted that when a stent causes injury to a coronary artery, it triggers a series of inflammatory reactions that result in the migration and proliferation of vascular smooth muscle cells (SMCs) within the vessel lumen and neointimal hyperplasia. In TCM, CAD is thought to be a result of "Qi deficiency and Blood Stasis". The severity of Blood Stasis syndrome is significantly correlated with the complexity of coronary lesions and the degree of stenosis and is an important factor affecting the occurrence of restenosis after PCI [57]. Many studies have demonstrated that Astragalus [58], salvia [59], tetramethylpyrazine [60], and Chuangxingol and paeoniflorin [61], which are main ingredients in TCM, play effective anti-inflammatory roles and inhibit SMC proliferation and migration. This may be the mechanism by which the above-mentioned CCMs prevent and treat restenosis.

Ideally, RCTs should adhere to known research design standards. In our examination of these studies, we did not have access to enough details related to these characteristics, although the baselines were comparable between comparison groups in the included studies. Details about randomization methodology were also lacking. In the 40 studies we reviewed, 11 reported that they implemented randomization using a random number table or a computer random number generator, such as SPSS software, and only 1 mentioned using sealed, opaque envelope concealment. Participants or outcome assessors were blinded in only 4 of the trials. In addition, studies that involve therapeutic trials should also report the rates of adverse reactions regardless of whether or not they occur. Reporting of adverse reactions is very important for evaluating the safety of interventional measures even though there is no guarantee that the adverse reactions were related to the interventional measure. Subgroup analyses showed that different types of Western medicines and drug forms affected outcomes. Furthermore, most of the 40 studies failed to mention the type of stent that was deployed (i.e., whether it was a bare metal stent or a drugeluting stent). Therefore, the difference in the curative effects observed between the CCM plus RWM and control groups is uncertain, and there is bias in our results. In our review, only 11 of the 40 included trials reported adverse reactions, rendering it difficult to systematically evaluate the safety of the evaluated CCMs in ISR. Future research on these topics will help to clarify the effectiveness and safety of CCMs in ISR.

\section{Conclusions}

Based on the results of this SR, we conclude combining RWM with CCMs may provide moderate efficacy in preventing ISR following PCI with stent placement. This is despite the fact that our investigation revealed the potential presence of bias in the identified studies. CCMs used to supplement Qi and activate the blood are suggested for preventing and treating restenosis. In addition, additional experimental studies should explore the mechanisms by which the main ingredients in CCM act to supplement Qi or activating the blood to prevent and treat restenosis. Future rigorously designed RCTs that explore CCM plus RWM therapies aimed at preventing post-PCI ISR should adhere to established design standards to overcome the limitations presented in this review. In particular, they should ensure adequate concealment of allocation and blinding of primary outcomes assessors.

\section{Conflicts of Interest}

The authors declare that there are no conflicts of interest regarding the publication of this paper.

\section{Authors' Contributions}

Qian Lin and Lu Liu conceived this SR. Lu Liu, Jing Liu, and Qun Gao drafted and revised this SR. Jinjin Lu, Jie Wan, 
Yan Li, Xiaoyun Cui, and Kun Zhou established the Data Management Committee and were separately responsible for study selection, data extraction, and risk of bias assessment procedures. Wenhao Jia, Yanchao Huang, and Wenbai Qu took charge of coordinating each process of this study, settling disagreements, and ensuring data safety. Qian Lin and Lu Liu contributed to the design of the statistical analyses. Lu Liu and Yang $\mathrm{Wu}$ were responsible for the overall data synthesis and analysis. Jing Liu contributed to polishing the language of the text. Qian Lin was responsible for the overall quality of this SR. All authors have read this manuscript and approved the publication of this SR.

\section{Acknowledgments}

This study is supported by the National Natural Science Foundation of China (no. 8160150703).

\section{References}

[1] E. D. Grech, "ABC of interventional cardiology: percutaneous coronary intervention. I: history and development," British Medical Journal, vol. 326, no. 7398, pp. 1080-1082, 2003.

[2] J. W. Moses, M. B. Leon, J. J. Popma et al., "Sirolimus-eluting stents versus standard stents in patients with stenosis in a native coronary artery," The New England Journal of Medicine, vol. 349, no. 14, pp. 1315-1323, 2003.

[3] L.-P. Zhao, W.-T. Xu, L. Wang et al., "Influence of insulin resistance on in-stent restenosis in patients undergoing coronary drug-eluting stent implantation after long-term angiographic follow-up," Coronary Artery Disease, vol. 26, no. 1, pp. 5-10, 2016.

[4] X. Wang, "Expert consensus on TCM diagnosis and treatment of chest pain after percutaneous coronary intervention (PCI)," Journal of Traditional Chinese Medicine, vol. 55, no. 13, pp. 11671170, 2014.

[5] Y. Ren, K.-J. Chen, and X.-M. Ruan, "Systematic review of randomized controlled trials on preventing and treating restenosis after percutaneous coronary intervention with Chinese medicine," Zhongguo Zhong Xi Yi Jie He Za Zhi Zhongguo Zhongxiyi Jiehe Zazhi, vol. 28, no. 7, pp. 597-601, 2008.

[6] G.-H. Zheng, J.-P. Liu, N. S. Wang, H.-Y. Chen, and J.-F. Chu, "Systematic review of chinese herbal medicines for preventing in-stent coronary restenosis after percutaneous coronary intervention," Evidence-Based Complementary and Alternative Medicine, vol. 2012, Article ID 253409, 19 pages, 2012.

[7] R. Xi, Q. Yang, K. Chen et al., "Chinese medicines for Activating Blood and Removing Blood Stasison the clinical outcome after percutaneous coronary intervention: a meta-analysis of randomized controlled trials," Cardiology (Switzerland), vol. 129, pp. 132-133, 2014.

[8] D. Moher, L. Shamseer, and M. Clarke, "Preferred reporting items for systematic review and meta-analysis protocols (PRISMA-P) 2015 statement," Systematic Reviews, vol. 4, no. 1, Article ID 1, 2015.

[9] J. P. T. Higgins and S. Green, "Cochrane handbook forsystematic reviews of interventions version 5.1.0 [updated March 2011], The Cochrane Collaboration," http://handbook.cochrane.org, 2011.

[10] Chinese Society of Cardiology of Chinese Medical Association, Chinese College of Cardiovascular, and Editorial Board of
Chinese Journal of Cardiology, "Chinese guidelines for percutaneous coronary intervention," Chinese Journal of Cardiology, vol. 44, no. 5, pp. 382-400, 2016.

[11] J. P. Higgins, D. G. Altman, and P. C. Gøtzsche, "The Cochrane collaboration's tool for assessing risk of bias in randomized trials," British Medical Journal, vol. 343, p. d5928, 2011.

[12] G. F. Dai, B. Z. zhang, and F. Wang, "Research of Tongmai decoction in the prevention and treatment of restenosis in patients with Qi Deficiency and Blood Stasis syndrome after percutaneous coronary intervention," Chinese Journal of Integrative Medicine on Cardio-/Cerebrovascular Disease, vol. 15, no. 2, pp. 136-142, 2017.

[13] R. Z. Zhangand and A. S. Gao, "Clinical observation on activating blood circulation and removing blood stasis medicine in the prevention and treatment of restenosis after coronary artery stenting," Guangming Journal of Chinese Medicine, vol. 32, no. 8, pp. 1083-1085, 2017.

[14] K. L. Wangand and Z. K. Yu, "Clinical effect of Huxinkang tablets in treatment of restenosis after percutaneous coronary interventionin patients with coronary heart disease," Hunan Journal of Traditional Chinese Medicine, vol. 32, no. 12, pp. 7-9, 2016.

[15] X. Y. Gong, "The effect of Huoxue tongmai fang in preventing coronary restenosis on ECG changes after coronary intervention," China Prescription Drug, vol. 14, no. 9, pp. 102-103, 2016.

[16] Y. Y. Tang and B. Fang, "Clinical observation of Yiqi Tongluo Huatan Decoction Combined with antiplatelet drugs for instent restenosis after percutaneous coronary intervention in patients with coronary heart disease," Zhejiang Journal of Traditional Chinese Medicine, vol. 51, no. 8, pp. 559-560, 2016.

[17] J. C. Zhou and M. J. Cheng, "Clinical effect analysis of integrated traditional Chinese and western medicine therapy on the prevention of restenosis (RS) after percutaneous coronary intervention (PCI)," Clinical Journal of Chinese Medicine, vol. 15, no. 8, pp. 29-31, 2016.

[18] H. B. Zhu, "Analysis of the efficacy of combined Statins with Mailuo shutong capsule in the prevention and treatment of restenosis in patients with coronary heart disease after PCI," China Journal of Chinese Materia Medica, pp. 1861-1862, 2015.

[19] N. H. Xiao, B. H. Liu, and Y. G. Zhang, "Clinical study of rosuvastatin combined with Xinxuetong Capsule in prevention of restenosis after coronary stent implantation," Journal of New Chinese Medicine, vol. 48, no. 3, pp. 25-27, 2016.

[20] D. D. Ren, H. L. Jia, Y. K. Guo et al., "Effects of Huoxue Tongmai Tang combined with western medicine to restenosis in patients after coronary artery stenting," Journal of Traditional Chinese Medicine, vol. 57, no. 4, pp. 311-315, 2016.

[21] Q. Z. Li, G. Hu, and Y. L. Wang, "Clinical observation of Guanxin Tongluo Capsule combined with dual antiplatelet drugs in prevention and treatment of in-stent restenosis of 34 patients with coronary heart disease after PCI," Guiding Journal of Traditional Chinese Medicine and Pharmacy, vol. 22, no. 3, pp. 80-82, 2016.

[22] Q. Y. Sun, "Efficacy of combination of traditional Chinese medicine and Western medicine in prevention and treatment of restenosis after coronary stent implantation," Journal of New Chinese Medicine, vol. 48, no. 2, pp. 31-33, 2016.

[23] M. T. Xu, Y. F. Chang, and X. Y. Wang, "Clinical observation of Guanxin suhe wan Combined with dual antiplatelet drugs in the Prevention and Treatment of Restenosis in in-stent restenosis after percutaneous coronary intervention," Guiding Journal of 
Traditional Chinese Medicine and Pharmacology, vol. 22, no. 2, pp. 71-73, 2016.

[24] X. L. Meng, "Clinical efficacy observation of replenishing Qi, activating blood circulation and removing toxics method on coronary restenosis after PCI," Clinical Journal of Chinese Medicine, vol. 8, no. 3, pp. 34-38, 2016.

[25] T. L. Yang, S. J. Pan, X. F. Zhang et al., "Effect of Tongxinluo capsule combined with pioglitazone for treatment of coronary stent restenosis in patients with coronary heart disease and type 2 diabetes: a prospective randomized double-blind placebocontrolled study," Chinese Journal of Integrated Traditional and Western Medicine in Intensive and Critical Care, vol. 23, no. 4, pp. 341-347, 2016.

[26] X. Wang, K. Y. Cheng, Z. W. Jia et al., "Clinical observation of coronary artery intervention for acute myocardial infarction with Huoxue Yiqi Tongmai Yin," Journal of Emergency in Traditional Chinese Medicine, vol. 25, no. 7, pp. 1415-1417, 2016.

[27] W. Zhou, X. B. Zhao, M. Zhou et al., "Effect of Longzhi dispensing granule on prevention and treatment of coronary restenosis after PCI," Journal of Tianjin Universityof Traditional Chinese Medicine, vol. 34, no. 5, pp. 265-269, 2015.

[28] W. D. Peng, B. Dong, and Y. Zhou, "Clinical observation on Mailuo shutong capsule combined with atorvastatin on restenosis of 32 patients with coronary artery disease after PCI," Guiding Journal of Traditional Chinese Medicine and Pharmacy, vol. 21, no. 18, pp. 69-71, 2015.

[29] Y. Zhang, D. S. Wang, and X. X. Gao, "Clinical research on preventing of No. 1 Xintong prescription on ISR," Guiding Journal of Traditional Chinese Medicine and Pharmacy, vol. 21, no. 15, pp. 26-27, 32, 2015.

[30] K. Wang, J. Zhang, and Y. J. Du, "Effects of Xuefuzhuyu capsules on the restenosis of AMI after percutaneous coronary intervention (PCI) in elderly patients," Practical Clinical Journal of Integrated Traditional Chinese and Western Medicine, vol. 15, no. 2, pp. 3-5, 2015.

[31] H. W. Lu, J. Zhang, X. Chen et al., "Clinical observation on Tongxinluo capsule combined with routine western medicine in the prevention of restenosis after percutaneous coronary intervention in 90 cases," Journal of Traditional Chinese Medicine, vol. 55, no. 24, pp. 2117-2110, 2014.

[32] P. F. Zhangand and H. C. Zhu, "Effect of Xinmai futong fang on restenosis after coronary stent implantation," Clinical Journal of Traditional Chinese Medicine, vol. 26, no. 11, pp. 1133-1134, 2014.

[33] Q. J. Shi, "Treatment of restenosis of 30 patients with coronary heart disease after PCI by Yiqi Huayu Jiedu decoction," Traditional Chinese Medicinal Research, vol. 27, no. 9, pp. 36-38, 2014.

[34] K. Chen, X. X. Gao, and Y. Zhang, "Clinical research on preventing of No. 2 Xintong prescription on ISR," Shandong Journal of Traditional Chinese Medicine, vol. 33, no. 4, pp. 290291, 2014.

[35] B. Li, "Effect of Qishen yiqi droplet on in-stent restenosis after coronary stent implantation," China Health Care \& Nutrition, vol. 5, pp. 2877-2878, 2014.

[36] C. W. Li, "Effects of qishen yiqi drop pill on coronary blood flow and left ventricular function in patients with anterior wall AMI after PCI," Chinese Journal of Integrative Medicine on Cardio-/ Cerebrovascular Disease, vol. 12, no. 12, pp. 1441-1443, 2014.

[37] Z. Q. Wang, "Clinical efficacy and mechanism analysis of TCM and western medicine therapy to prevent percutaneous coronary intervention (PCI) restenosis (RS)," Chinese Archives of Traditional Chinese Medicine, vol. 31, no. 12, pp. 2819-2822, 2013.
[38] Q. Zhang, S. K. Liu, Y. X. Li et al., "The effect of Shenshao oral lotion to restenosis after percutaneous coronary intervention," Chinese Journal of Arteriosclerosis, vol. 21, no. 10, pp. 903-906, 2013.

[39] X. D. Deng, "Effect of Tongxinluo Capsuleon restenosis after PCI in patients with CHD," China Medicine and Phamacy, vol. 3, no. 12, pp. 67-68, 2013.

[40] K. Chen, X. X. Gao, and Y. Zhang, "Clinical research on preventing of No. 2 Xintong prescription on ISR," Journal of Sichuan of Traditional Chinese Medicine, vol. 31, no. 11, pp. 76-77, 2013.

[41] X. C. Si and Y. P. Jia, "Effect of Shexiang Baoxin pellet on longterm prognosis in patients with coronary disease under PCI," Clinical Journal of Chinese Medicine, vol. 5, no. 1, pp. 5-6, 2013.

[42] P. Han, "Clinical observation of Gualou Xiebai Banxia decoction combined with Xuefu Zhuyu decoction in prevention and treatment coronary artery in-stent restenosis," Chinese Medicine, vol. 28, no. 26, 172 pages, 2012.

[43] X. Y. Ren, X. X. Gao, K. Chen et al., "Clinical observation of supplementing Qi and activating blood stasismethod combined with western medicine in prevention of restenosis after coronary stent implantation," Journal of Sichuan of Traditional Chinese Medicine, vol. 30, no. 9, pp. 68-69, 2012.

[44] X. Y. Niu, Y. Zhao, X. S. Zhang et al., "Intervention with Jingqi Danshen decoction on restenosis of 41 patients with coronary artery disease after PCI," Journal of Traditional Chinese Medicine, vol. 53, no. 17, pp. 1503-1504, 2012.

[45] X. X. Gao and Y. Zhang, "Clinical observation of No. 1 Xintong Prescription in prevention and treatment of restenosis after percutaneous coronary intervention in 30 cases," Hebei Journal of Traditional Chinese Medicine, vol. 34, no. 6, pp. 832-833, 2012.

[46] G. F. Dai and S. J. Yang, "Clinical observation of Tongxinluo capsulein the treatment of restenosis after PCI," Guangming Journal of Chinese Medicine, vol. 26, no. 9, pp. 1823-1824, 2011.

[47] C. L. Mei, Y. Xu, and X. Q. Shi, "Blood Stasisparty to the joint western medicine control percutaneous coronary angioplasty restenosis after 61 cases clinical observation," Guiding Journal of Traditional Chinese Medicine and Pharmacy, vol. 17, no. 6, pp. 35-36, 2011.

[48] X. D. Wang and B. Lou, "Effect of Tongxinluo capsule on restenosis after coronary artery stent implantation in patients with type-2 diabetes mellitus," Journal of Nanjing University of Traditional Chinese Medicine, vol. 26, no. 6, pp. 464-467, 2010 (Chinese).

[49] S. J. Cheng, H. B. Fang, J. S. He, and etal., "Effect of Anxin granule on the restenosis after percutaneous coronary intervention," Journal of Traditional Chinese Medicine, vol. 51, no. 7, pp. 603606, 2010.

[50] G. F. Dai, H. B. Yang, and T. H. Du, "Intervention of Yiqi Huoxue Tongmai decoction on restenosis of 49 cases with coronary artery disease after PCI," Liaoning Journal of Traditional Chinese Medicine, vol. 37, no. 6, pp. 1082-1083, 2010 (Chinese).

[51] L. L. Liu and P. Y. Zhang, "Clinical study on prevention and treatment of restenosis after coronary intervention by Jingtian zaitong capsule," Jilin Journal of Traditional Chinese Medicine, vol. 30, no. 1, pp. 35-36, 2010.

[52] The Joint International Society and Federation of Cardiology/ World Health Organization task force, "Nomenclature and criteria for diagnosis of ischemic heart disease," Circulation, vol. 59, no. 3, pp. 607-609, 1979. 
[53] R. E. Kuntz and D. S. Baim, "Defining coronary restenosis: newer clinical and angiographic paradigms," Circulation, vol. 88, no. 3, pp. 1310-1323, 1993.

[54] L. Mauri, E. J. Orav, A. J. O’Malley et al., “Relationship of late loss in lumen diameter to coronary restenosis in sirolimus-eluting stents," Circulation, vol. 111, no. 3, pp. 321-327, 2005.

[55] K. M. Lin, D. H. Heand, and W. H. Li, "Impact of stent overexpansion at distal edge: insights from a 12-month followup study," European Review for Medical and Pharmacological Sciences, vol. 19, no. 10, pp. 1874-1880, 2015.

[56] T. Kimura, H. Yokoi, Y. Nakagawa et al., "Three-year follow-up after implantation of metallic coronary-artery stents," The New England Journal of Medicine, vol. 334, no. 9, pp. 561-566, 1996.

[57] G. Yu and J. Wang, "Blood stasis syndrome of coronary heart disease: a perspective of modern medicine," Chinese Journal of Integrative Medicine, vol. 20, no. 4, pp. 300-306, 2014.

[58] S. Ohkawara, Y. Okuma, T. Uehara, T. Yamagishi, and Y. Nomura, "Astrapterocarpan isolated from Astragalus membranaceus inhibits proliferation of vascular smooth muscle cells," European Journal of Pharmacology, vol. 525, no. 1-3, pp. 41-47, 2005.

[59] L. Sun, R. Zhao, L. Zhang et al., "Salvianolic acid A inhibits PDGF-BB induced vascular smooth muscle cell migration and proliferation while does not constrain endothelial cell proliferation and nitric oxide biosynthesis," Molecules, vol. 17, no. 3, pp. 3333-3347, 2012.

[60] G. Ma, S. Ding, Y. Feng, C. Shen, L. Chen, and Z. Chen, "Tetramethylpyrazine-eluting stents prevented in-stent restenosis in a porcine model," Journal of Cardiovascular Pharmacology, vol. 50, no. 2, pp. 201-205, 2007.

[61] H. Xu, D. Shi, and K. Chen, "Inhibition of vascular remodelling in a porcine coronary injury model by herbal extract XS0601," Chinese Medicine, 2006, http://www.cmjournal.org/content/1/1/2. 


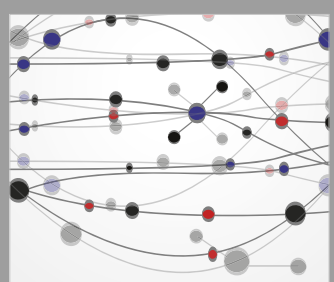

The Scientific World Journal
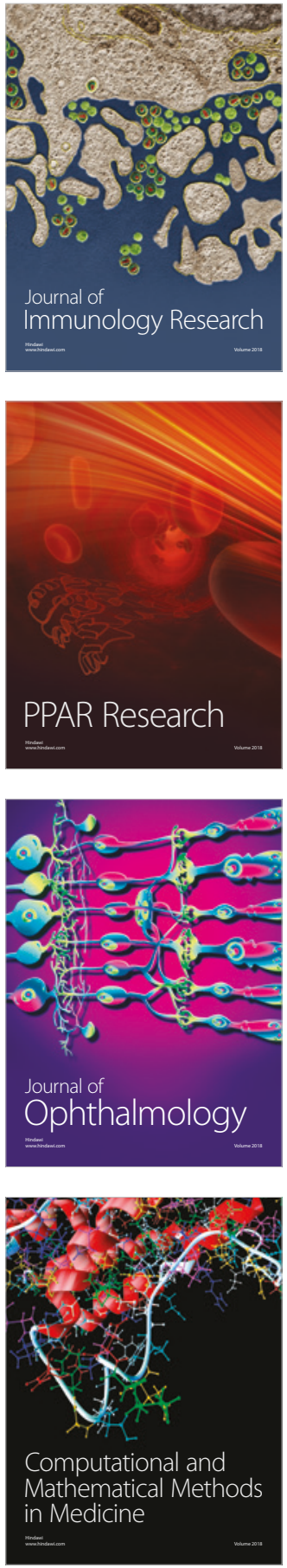

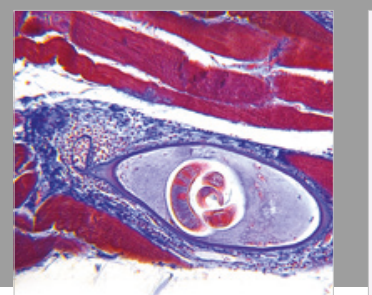

Gastroenterology Research and Practice

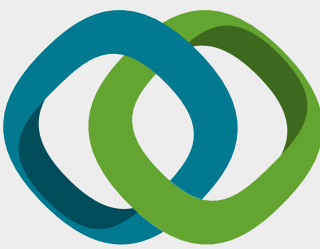

\section{Hindawi}

Submit your manuscripts at

www.hindawi.com
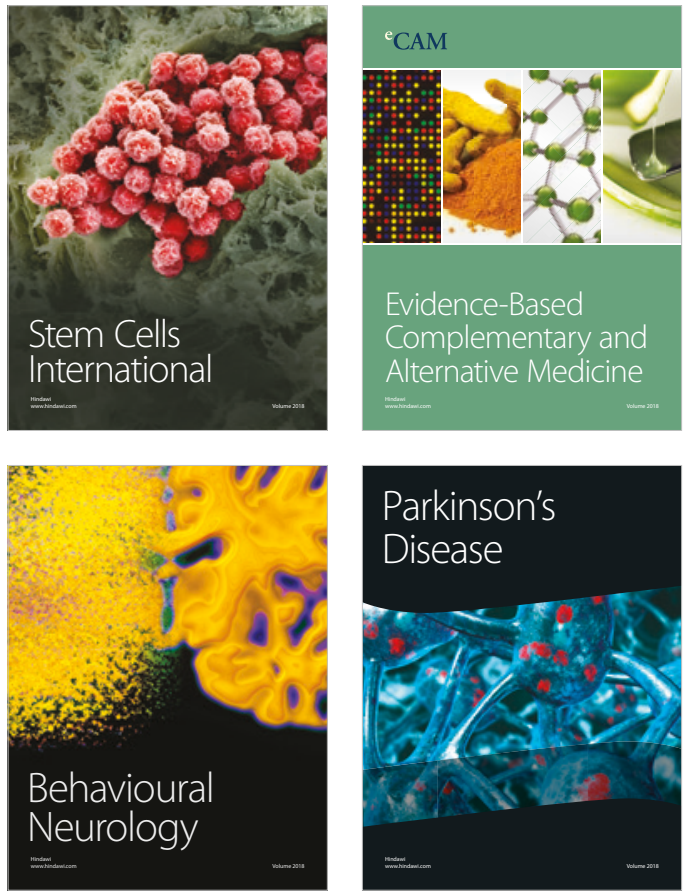

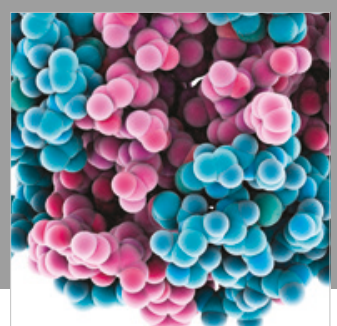

ournal of

Diabetes Research

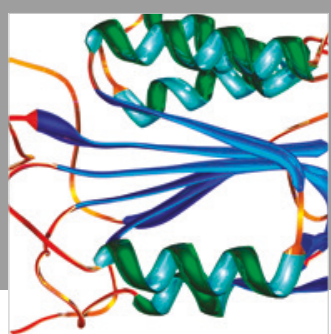

Disease Markers
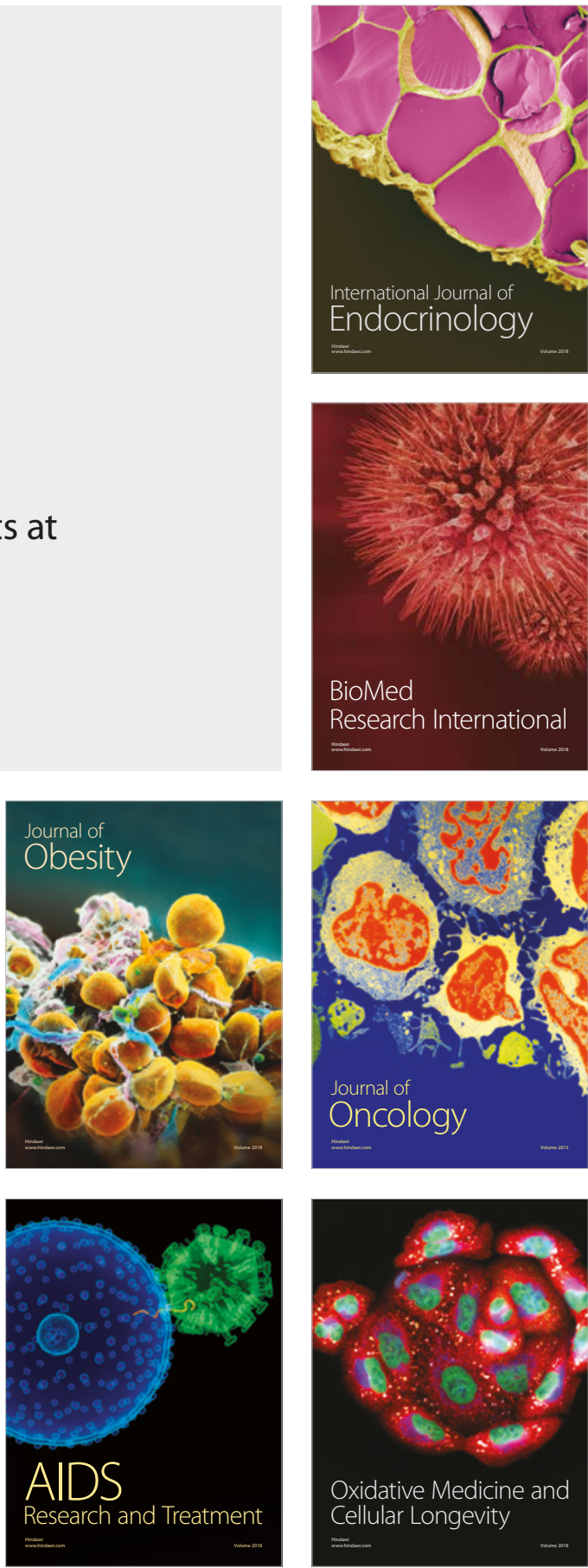